\title{
EXISTENCE AND UNIFORM BOUNDEDNESS OF OPTIMAL SOLUTIONS OF VARIATIONAL PROBLEMS
}

\author{
ALEXANDER J. ZASLAVSKI
}

\begin{abstract}
Given an $x_{0} \in R^{n}$ we study the infinite horizon problem of minimizing the expression $\int_{0}^{T} f\left(t, x(t), x^{\prime}(t)\right) d t$ as $T$ grows to infinity where $x:[0, \infty) \rightarrow R^{n}$ satisfies the initial condition $x(0)=x_{0}$. We analyse the existence and the properties of approximate solutions for every prescribed initial value $x_{0}$. We also establish that for every bounded set $E \subset R^{n}$ the $C([0, T])$ norms of approximate solutions $x:[0, T] \rightarrow R^{n}$ for the minimization problem on an interval $[0, T]$ with $x(0), x(T) \in E$ are bounded by some constant which does not depend on $T$.
\end{abstract}

\section{INTRODUCTION}

The study of variational and optimal control problems defined on infinte intervals has recently been a rapidly growing area of research. These problems arise in engineering (see Anderson and Moore [1], Artstein and Leizarowitz [2]), in models of economic growth (see Rockafellar [14], Zaslavski [20]), in infinite discrete models of solid-state physics related to dislocations in one-dimensional crystals which are under discussion in Aubry and Le Daeron [3], Zaslavski [16] and in the theory of thermodynamical equilibrium of materials (see Leizarowitz and Mizel [12], Coleman, Marcus and Mizel [7], Zaslavski [17,18]).

We consider the infinite horizon problem of minimizing the expression

$$
\int_{0}^{T} f\left(t, x(t), x^{\prime}(t)\right) d t
$$

as $T$ grows to infinity where a function $x:[0, \infty) \rightarrow K$ is absolutely continuous (a.c.) and satisfies the initial condition $x(0)=x_{0}, K \subset R^{n}$ is a

1991 Mathematics Subject Classification. 49J99, 58F99.

Key words and phrases. Infinite horizon, overtaking optimal function, good function.

Received: December 9, 1996. 
closed convex set and $f$ belongs to a complete metric space of functions to be described below.

The following notion known as the overtaking optimality criterion was introduced in the economics literature by Gale [8] and von Weizsacker [15] and has been used in control theory by Artstein and Leizarowitz [2], Brock and Haurie [5], Carlson [6] and Leizarowitz [11].

An a.c. function $x:[0, \infty) \rightarrow K$ is called $(f)$-overtaking optimal if for any a.c. function $y:[0, \infty) \rightarrow K$ satisfying $y(0)=x(0)$

$$
\limsup _{T \rightarrow \infty} \int_{0}^{T}\left[f\left(t, x(t), x^{\prime}(t)\right)-f\left(t, y(t), y^{\prime}(t)\right)\right] d t \leq 0 .
$$

Usually it is difficult to establish the existence of overtaking optimal solutions, and actually, in general they may fail to exist. Most studies that are concerned with their existence assume convex integrands ([11], [5],[14]).

Another type of optimality criterion for infinite horizon problems (which is probably the weakest optimality concept) was introduced by Aubry and Le Daeron [3] in their study of the discrete Frenkel-Kontorova model related to dislocations in one-dimensional crystals. More recently this optimality criterion was used by Moser [13], Leizarowitz and Mizel [12] and Zaslavski [16]. A similar notion was introduced in Halkin [9] for his proof of the maximum principle.

Let $I$ be either $[0, \infty)$ or $(-\infty, \infty)$. An a.c. function $x: I \rightarrow K$ is called an $(f)$-minimal solution if for each $T_{1} \in I, T_{2}>T_{1}$ and each a.c. function $y:\left[T_{1}, T_{2}\right] \rightarrow K$ which satisfies $y\left(T_{i}\right)=x\left(T_{i}\right), i=1,2$ the following relation holds:

$$
\int_{T_{1}}^{T_{2}}\left[f\left(t, x(t), x^{\prime}(t)\right)-f\left(t, y(t), y^{\prime}(t)\right)\right] d t \leq 0 .
$$

Clearly every $(f)$-overtaking optimal function is an $(f)$-minimal solution.

In the present paper we consider a functional space of integrands $\mathfrak{M}$ described in Section 1 and analyze existence and properties of $(f)$-minimal solutions with $f \in \mathfrak{M}$. More exactly we will show that given $f \in \mathfrak{M}$ and $z \in R^{n}$ there exists a bounded $(f)$-minimal solution $Z:[0, \infty) \rightarrow R^{n}$ satisfying $Z(0)=z$ such that any other a.c. function $Y:[0, \infty) \rightarrow R^{n}$ is not "better" then $Z$. We will also establish that given $f \in \mathfrak{M}$ and a bounded set $E \subset R^{n}$ the $C([0, T])$ norms of approximate solutions $x:[0, T] \rightarrow R^{n}$ for the minimization problem on an interval $[0, T]$ with $x(0), x(T) \in E$ are bounded by some constant which depends only on $f$ and $E$. These results which are valid for any $f \in \mathfrak{M}$ have been applied in [19] to get more information about the existence of optimal solutions over an infinite horizon and about the structure of optimal solutions on finite intervals for a generic integrand $f \in \mathfrak{M}$.

The paper is organized as follows. In Section 1 we state our main theorems, Section 2 contains several preliminary results, in Section 3 we consider discrete-time control systems obtained by discretization of variational problems and in Section 4 we prove the main theorems. 


\section{Statements of MAin Results}

Let $K \subset R^{n}$ be a closed convex set. Denote by $|\cdot|$ the Euclidean norm in $R^{n}$ and denote by $\mathfrak{M}$ the set of continuous functions $f:[0, \infty) \times K \times R^{n} \rightarrow$ $R^{1}$ which satisfy the following assumptions:

(A) (i) for each $(t, x) \in[0, \infty) \times K$ the function $f(t, x, \cdot): R^{n} \rightarrow R^{1}$ is convex;

(ii) the function $f$ is bounded on $[0, \infty) \times E$ for any bounded set $E \subset$ $K \times R^{n}$;

(iii) $f(t, x, u) \geq \sup \{\psi(|x|), \psi(|u|)|u|\}-a$ for each $(t, x, u) \in[0, \infty) \times K \times$ $R^{n}$ where $a>0$ is a constant and $\psi:[0, \infty) \rightarrow[0, \infty)$ is an increasing function such that $\psi(t) \rightarrow \infty$ as $t \rightarrow \infty$ (here $a$ and $\psi$ are independent on $f)$;

(iv) for each $M, \epsilon>0$ there exist $\Gamma, \delta>0$ such that

$$
\left|f\left(t, x_{1}, u_{1}\right)-f\left(t, x_{2}, u_{2}\right)\right| \leq \epsilon \sup \left\{f\left(t, x_{1}, u_{1}\right), f\left(t, x_{2}, u_{2}\right)\right\}
$$

for each $t \in[0, \infty)$, each $u_{1}, u_{2} \in R^{n}$ and each $x_{1}, x_{2} \in K$ which satisfy

$$
\left|x_{i}\right| \leq M,\left|u_{i}\right| \geq \Gamma, i=1,2, \quad \sup \left\{\left|x_{1}-x_{2}\right|,\left|u_{1}-u_{2}\right|\right\} \leq \delta
$$

(v) for each $M, \epsilon>0$ there exist $\delta>0$ such that

$$
\left|f\left(t, x_{1}, u_{1}\right)-f\left(t, x_{2}, u_{2}\right)\right| \leq \epsilon
$$

for each $t \in[0, \infty)$, each $u_{1}, u_{2} \in R^{n}$ and each $x_{1}, x_{2} \in K$ which satisfy

$$
\left|x_{i}\right|,\left|u_{i}\right| \leq M, i=1,2, \quad \sup \left\{\left|x_{1}-x_{2}\right|, \mid u_{1}-u_{2}\right\} \leq \delta .
$$

When $K=R^{n}$ it is an elementary exercise to show that an integrand $f=f(t, x, u) \in C^{1}\left([0, \infty) \times R^{n} \times R^{n}\right)$ belongs to $\mathfrak{M}$ if $f$ satisfies assumptions (Ai), (Aiii) with a constant $a>0$ and a function $\psi:[0, \infty) \rightarrow[0, \infty)$,

$$
\sup \{|f(t, 0,0)|: t \in[0, \infty)\}<\infty
$$

and there exists an increasing function $\psi_{0}:[0, \infty) \rightarrow[0, \infty)$ such that

$$
\sup \{|\partial f / \partial x(t, x, u)|,|\partial f / \partial u(t, x, u)|\} \leq \psi_{0}(|x|)(1+\psi(|u|)|u|)
$$

for each $t \in[0, \infty), x, u \in R^{n}$.

For the set $\mathfrak{M}$ we consider the uniformity which is determined by the the following base

$$
\begin{gathered}
E(N, \epsilon, \lambda)=\{(f, g) \in \mathfrak{M} \times \mathfrak{M}:|f(t, x, u)-g(t, x, u)| \leq \epsilon \\
\left(t \in[0, \infty), u \in R^{n}, x \in K,|x|,|u| \leq N\right),
\end{gathered}
$$




$$
\begin{gathered}
(|f(t, x, u)|+1)(|g(t, x, u)|+1)^{-1} \in\left[\lambda^{-1}, \lambda\right] \\
\left.\left(t \in[0, \infty), u \in R^{n}, x \in K,|x| \leq N\right)\right\}
\end{gathered}
$$

where $N>0, \epsilon>0, \lambda>1$.

Clearly, the uniform space $\mathfrak{M}$ is Hausdorff and has a countable base. Therefore $\mathfrak{M}$ is metrizable. We will show that the uniform space $\mathfrak{M}$ is complete (see Proposition 2.2).

We consider functionals of the form

$$
I^{f}\left(T_{1}, T_{2}, x\right)=\int_{T_{1}}^{T_{2}} f\left(t, x(t), x^{\prime}(t)\right) d t
$$

where $f \in \mathfrak{M}, 0 \leq T_{1}<T_{2}<\infty$ and $x:\left[T_{1}, T_{2}\right] \rightarrow K$ is an a.c. function.

For $f \in \mathfrak{M}, a, b \in K$ and numbers $T_{1}, T_{2}$ satisfying $0 \leq T_{1}<T_{2}$ we set

$$
U^{f}\left(T_{1}, T_{2}, a, b\right)=\inf \left\{I^{f}\left(T_{1}, T_{2}, x\right): x:\left[T_{1}, T_{2}\right] \rightarrow K\right.
$$

is an a.c. function satisfying $\left.x\left(T_{1}\right)=a, x\left(T_{2}\right)=b\right\}$,

$$
\sigma^{f}\left(T_{1}, T_{2}, a\right)=\inf \left\{U^{f}\left(T_{1}, T_{2}, a, b\right): b \in K\right\}
$$

It is easy to see that $-\infty<U^{f}\left(T_{1}, T_{2}, a, b\right)<\infty$ for each $f \in \mathfrak{M}$, each $a, b \in K$ and each numbers $T_{1}, T_{2}$ satisfying $0 \leq T_{1}<T_{2}$.

Here we follow Leizarowitz [10] in defining "good functions" for the variational problem.

Let $f \in \mathfrak{M}$. An a.c. function $x:[0, \infty) \rightarrow K$ is called an $(f)$-good function if for any a.c. function $y:[0, \infty) \rightarrow K$ there is a number $M_{y}$ such that

$$
I^{f}(0, T, y) \geq M_{y}+I^{f}(0, T, x) \text { for each } T \in(0, \infty) .
$$

In this paper our goal will be to study the set of $(f)$-good functions. We will establish the following results.

Theorem 1.1. For each $f \in \mathfrak{M}$ and each $z \in K$ there exists an $(f)$-good function $Z^{f}:[0, \infty) \rightarrow K$ satisfying $Z^{f}(0)=z$ such that:

1. For each $f \in \mathfrak{M}$, each $z \in K$ and each a.c. function $y:[0, \infty) \rightarrow K$ one of the following properties holds:

(i) $I^{f}(0, T, y)-I^{f}\left(0, T, Z^{f}\right) \rightarrow \infty$ as $T \rightarrow \infty$;

(ii) $\sup \left\{\left|I^{f}(0, T, y)-I^{f}\left(0, T, Z^{f}\right)\right|: T \in(0, \infty)\right\}<\infty$,

$$
\sup \{|y(t)|: t \in[0, \infty)\}<\infty .
$$

2. For each $f \in \mathfrak{M}$ and each number $M>\inf \{|u|: u \in K\}$ there exist a neighborhood $U$ of $f$ in $\mathfrak{M}$ and a number $Q>0$ such that

$$
\sup \left\{\left|Z^{g}(t)\right|: t \in[0, \infty)\right\} \leq Q
$$


for each $g \in U$ and each $z \in K$ satisfying $|z| \leq M$.

3. For each $f \in \mathfrak{M}$ and each number $M>\inf \{|u|: u \in K\}$ there exist a neighborhood $U$ of $f$ in $\mathfrak{M}$ and a number $Q>0$ such that for each $g \in U$, each $z \in K$ satisfying $|z| \leq M$, each $T_{1} \geq 0, T_{2}>T_{1}$ and each a.c. function $y:\left[T_{1}, T_{2}\right] \rightarrow K$ satisfying $\left|y\left(T_{1}\right)\right| \leq M$ the following relation holds:

$$
I^{g}\left(T_{1}, T_{2}, Z^{g}\right) \leq I^{g}\left(T_{1}, T_{2}, y\right)+Q
$$

4. If $K=R^{n}$ then for each $f \in \mathfrak{M}$ and each $z \in R^{n}$ the function $Z^{f}:[0, \infty) \rightarrow R^{n}$ is an $(f)$-minimal solution.

Corollary 1.1. Let $f \in \mathfrak{M}, z \in K$ and let $y:[0, \infty) \rightarrow K$ be an a.c. function. Then $y$ is an $(f)$-good function if and only if condition (ii) of Assertion 1 holds.

Theorem 1.2. For each $f \in \mathfrak{M}$ there exists a neighborhood $U$ of $f$ in $\mathfrak{M}$ and a number $M>0$ such that for each $g \in U$ and each $(g)$-good function $x:[0 \infty) \rightarrow K$

$$
\limsup _{t \rightarrow \infty}|x(t)|<M
$$

In this paper we prove the following result which establishes that for every bounded set $E \subset K$ the $C([0, T])$ norms of approximate solutions $x:[0, T] \rightarrow K$ for the minimization problem on an interval $[0, T]$ with $x(0), x(T) \in E$ are bounded by some constant which does not depend on $T$.

Theorem 1.3. Let $f \in \mathfrak{M}$ and $M_{1}, M_{2}, c$ be positive numbers. Then there exist a neighborhood $U$ of $f$ in $\mathfrak{M}$ and a number $S>0$ such that for each $g \in U$, each $T_{1} \in[0, \infty)$ and each $T_{2} \in\left[T_{1}+c, \infty\right)$ the following properties hold:

(i) for each $x, y \in K$ satisfying $|x|,|y| \leq M_{1}$ and each a.c. function $v:\left[T_{1}, T_{2}\right] \rightarrow K$ satisfying

$$
v\left(T_{1}\right)=x, v\left(T_{2}\right)=y, I^{g}\left(T_{1}, T_{2}, v\right) \leq U^{g}\left(T_{1}, T_{2}, x, y\right)+M_{2}
$$

the following relation holds:

$$
|v(t)| \leq S, t \in\left[T_{1}, T_{2}\right]
$$

(ii) for each $x \in K$ satisfying $|x| \leq M_{1}$ and each a.c. function $v$ : $\left[T_{1}, T_{2}\right] \rightarrow K$ satisfying

$$
v\left(T_{1}\right)=x, I^{g}\left(T_{1}, T_{2}, v\right) \leq \sigma^{g}\left(T_{1}, T_{2}, x\right)+M_{2}
$$

relation (1.6) holds. 


\section{Preliminary Results}

Proposition 2.1. Let $f \in \mathfrak{M}, M$ and $\epsilon$ be positive numbers. Then there exist $\Gamma, \delta>0$ such that

$$
\left|f\left(t, x_{1}, u_{1}\right)-f\left(t, x_{2}, u_{2}\right)\right| \leq \epsilon \inf \left\{f\left(t, x_{1}, u_{1}\right), f\left(t, x_{2}, u_{2}\right)\right\}
$$

for each $t \in[0, \infty)$, each $u_{1}, u_{2} \in R^{n}$ and each $x_{1}, x_{2} \in K$ which satisfy

$$
\left|x_{i}\right| \leq M,\left|u_{i}\right| \geq \Gamma, i=1,2,\left|u_{1}-u_{2}\right|,\left|x_{1}-x_{2}\right| \leq \delta .
$$

Proof. Fix a number

$$
\epsilon_{0} \in\left(0,4^{-1} \inf \{1, \epsilon\}\right)
$$

By Assumption (Aiv) there exist $\Gamma, \delta>0$ such that

$$
\left|f\left(t, x_{1}, u_{1}\right)-f\left(t, x_{2}, u_{2}\right)\right| \leq \epsilon_{0} \sup \left\{f\left(t, x_{1}, u_{1}\right), f\left(t, x_{2}, u_{2}\right)\right\}
$$

for each $t \in[0, \infty)$, each $u_{1}, u_{2} \in R^{n}$ and each $x_{1}, x_{2} \in K$ which satisfy (2.1)

Assume that $t \in[0, \infty), u_{1}, u_{2} \in R^{n}$ and $x_{1}, x_{2} \in K$ satisfy (2.1). It follows from the definition of $\Gamma, \delta$ and (2.2), (2.3) that

$$
\begin{aligned}
& \inf \left\{f\left(t, x_{1}, u_{1}\right), f\left(t, x_{2}, u_{2}\right)\right\} \geq\left(1-\epsilon_{0}\right) \sup \left\{f\left(t, x_{1}, u_{1}\right), f\left(t, x_{2}, u_{2}\right)\right\} \\
\geq & \left(1-\epsilon_{0}\right) \epsilon_{0}^{-1}\left|f\left(t, x_{1}, u_{1}\right)-f\left(t, x_{2}, u_{2}\right)\right| \geq \epsilon^{-1}\left|f\left(t, x_{1}, u_{1}\right)-f\left(t, x_{2}, u_{2}\right)\right| .
\end{aligned}
$$

The proposition is proved.

Proposition 2.2. The uniform space $\mathfrak{M}$ is complete.

Proof. Assume that $\left\{f_{i}\right\}_{i=1}^{\infty} \subset \mathfrak{M}$ is a Cauchy sequence. Clearly, there exists a function $f:[0, \infty) \times K \times R^{n} \rightarrow R^{1}$ such that for each $(t, x, u) \in$ $[0, \infty) \times K \times R^{n}$

$$
f(t, x, u)=\lim _{i \rightarrow \infty} f_{i}(t, x, u) .
$$

To prove the proposition it is sufficient to show that $f$ satisfies Assumption (Aiv).

Let $M, \epsilon$ be positive numbers. Fix a number $\lambda>1$ such that

$$
\lambda^{2}-1<8^{-1} \epsilon \text {. }
$$

Clearly there exists an integer $j \geq 1$ such that

$$
\left(f_{i}, f_{j}\right) \in E(M, \epsilon, \lambda) \text { for any integer } i \geq j \text {. }
$$


By (2.5) and the properties of $\psi$ there exists a number $\Gamma_{0}$ such that

$$
\Gamma_{0}>1, \psi\left(\Gamma_{0}\right) \geq 2 a, \lambda^{2}\left(1+2 \psi\left(\Gamma_{0}\right)^{-1}\right)^{2}-1<8^{-1} \epsilon
$$

Fix a positive number $\epsilon_{1}$ which satisfies

$$
8 \epsilon_{1}\left[\lambda\left(1+2 \psi\left(\Gamma_{0}\right)^{-1}\right)\right]^{2}<\epsilon .
$$

By Proposition 2.1 there exist numbers $\Gamma, \delta>0$ such that (2.9)

$$
\Gamma>\Gamma_{0},\left|f_{j}\left(t, x_{1}, u_{1}\right)-f_{j}\left(t, x_{2}, u_{2}\right)\right| \leq \epsilon_{1} \inf \left\{f_{j}\left(t, x_{1}, u_{1}\right), f_{j}\left(t, x_{2}, u_{2}\right)\right\}
$$

for each $t \in[0, \infty)$, each $u_{1}, u_{2} \in R^{n}$ and each $x_{1}, x_{2} \in K$ which satisfy (2.1).

Assume that $t \in[0, \infty), u_{1}, u_{2} \in R^{n}, x_{1}, x_{2} \in K$ satisfy (2.1). It follows from the definition of $\Gamma, \delta$ that (2.9) holds. By (1.1), (2.4), (2.6) and (2.1)

$$
\left(\left|f\left(t, x_{i}, u_{i}\right)\right|+1\right)\left(\left|f_{j}\left(t, x_{i}, u_{i}\right)\right|+1\right)^{-1} \in\left[\lambda^{-1}, \lambda\right], i=1,2 .
$$

Assumption (Aiii), (2.1), (2.7) and (2.9) imply that

$$
\inf \left\{f\left(t, x_{i}, u_{i}\right), f_{j}\left(t, x_{i}, u_{i}\right)\right\} \geq 2^{-1} \psi\left(\Gamma_{0}\right), i=1,2 .
$$

Together with (2.10) this implies that

$f\left(t, x_{i}, u_{i}\right) f_{j}\left(t, x_{i}, u_{i}\right)^{-1} \in\left[\left(\lambda\left(1+2 \psi\left(\Gamma_{0}\right)^{-1}\right)\right)^{-1}, \lambda\left(1+2 \psi\left(\Gamma_{0}\right)^{-1}\right)\right], i=1,2$.

We may assume without loss of generality that

$$
f\left(t, x_{1}, u_{1}\right) \geq f\left(t, x_{2}, u_{2}\right)
$$

It follows from (2.12), (2.9), (2.8) and (2.7) that

$$
\begin{gathered}
f\left(t, x_{1}, u_{1}\right)-f\left(t, x_{2}, u_{2}\right) \leq \lambda\left(1+2 \psi\left(\Gamma_{0}\right)^{-1}\right) f_{j}\left(t, x_{1}, u_{1}\right) \\
-\left(\lambda\left(1+2 \psi\left(\Gamma_{0}\right)^{-1}\right)\right)^{-1} f_{j}\left(t, x_{2}, u_{2}\right)=\lambda\left(1+2 \psi\left(\Gamma_{0}\right)^{-1}\right)\left[f_{j}\left(t, x_{1}, u_{1}\right)-\right. \\
\left.f_{j}\left(t, x_{2}, u_{2}\right)\right]+f_{j}\left(t, x_{2}, u_{2}\right)\left[\lambda\left(1+2 \psi\left(\Gamma_{0}\right)^{-1}\right)-\left(\lambda\left(1+2 \psi\left(\Gamma_{0}\right)^{-1}\right)\right)^{-1}\right] \\
\leq \lambda\left(1+2 \psi\left(\Gamma_{0}\right)^{-1}\right) \epsilon_{1} f_{j}\left(t, x_{2}, u_{2}\right)+f_{j}\left(t, x_{2}, u_{2}\right)\left[\lambda\left(1+2 \psi\left(\Gamma_{0}\right)^{-1}\right)-\right. \\
\left.\left(\lambda\left(1+2 \psi\left(\Gamma_{0}\right)^{-1}\right)\right)^{-1}\right] \leq \epsilon_{1}\left[\lambda\left(1+2 \psi\left(\Gamma_{0}\right)^{-1}\right)\right]^{2} f\left(t, x_{2}, u_{2}\right)+ \\
f\left(t, x_{2}, u_{2}\right)\left[\lambda^{2}\left(1+2 \psi\left(\Gamma_{0}\right)^{-1}\right)^{2}-1\right] \leq \epsilon f\left(t, x_{2}, u_{2}\right) .
\end{gathered}
$$

Therefore the function $f$ satisfies Assumption (Aiv). This completes the proof of the proposition. 
Proposition 2.3. Let $M_{1}>0,0<\tau_{0}<\tau_{1}$. Then there exists a number $M_{2}>0$ such that for each $f \in \mathfrak{M}$, each pair of numbers $T_{1}, T_{2}$ satisfying

$$
0 \leq T_{1}<T_{2}, T_{2}-T_{1} \in\left[\tau_{0}, \tau_{1}\right]
$$

and each a.c. function $x:\left[T_{1}, T_{2}\right] \rightarrow K$ which satisfies

$$
I^{f}\left(T_{1}, T_{2}, x\right) \leq M_{1}
$$

the following relation holds:

$$
|x(t)| \leq M_{2}, t \in\left[T_{1}, T_{2}\right] .
$$

Proof. By Assumption (Aiii) and the properties of the function $\psi$ there exists a number $c_{0}>0$ such that

$$
f(t, x, u) \geq|u|
$$

for each $f \in \mathfrak{M}$ and each $(t, x, u) \in[0, \infty) \times K \times R^{n}$ satisfying $|u| \geq c_{0}$, and

$$
f(t, x, u) \geq 2 M_{1}\left(\inf \left\{1, \tau_{0}\right\}\right)^{-1}
$$

for each $f \in \mathfrak{M}$ and each $(t, x, u) \in[0, \infty) \times K \times R^{n}$ satisfying $|x| \geq c_{0}$. Fix a number

$$
M_{2}>1+M_{1}+a \tau_{1}+c_{0}\left(1+\tau_{1}\right)
$$

(recall $a$ in Asssumption (Aiii)).

Let $f \in \mathfrak{M}, T_{1}, T_{2}$ be numbers satisfying (2.14) and let $x:\left[T_{1}, T_{2}\right] \rightarrow K$ be an a.c. function satisfying (2.15). We will show that (2.16) holds.

Assume the contrary. Then there exists $t_{0} \in\left[T_{1}, T_{2}\right]$ such that

$$
\left|x\left(t_{0}\right)\right|>M_{2} \text {. }
$$

By the definition of $c_{0},(2.18),(2.14)$ and $(2.15)$ there exists $t_{1} \in\left[T_{1}, T_{2}\right]$ satisfying

$$
\left|x\left(t_{1}\right)\right| \leq c_{0}
$$

Set

$$
E=\left[\inf \left\{t_{0}, t_{1}\right\}, \sup \left\{t_{0}, t_{1}\right\}\right], E_{1}=\left\{t \in E:\left|x^{\prime}(t)\right| \geq c_{0}\right\}, E_{2}=E \backslash E_{1} .
$$

It follows from $(2.22),(2.14)$, the definition of $c_{0},(2.17)$, Assumption (Aiii) and (2.15) that

$$
\begin{gathered}
\left|x\left(t_{1}\right)-x\left(t_{0}\right)\right| \leq \int_{E_{1}}\left|x^{\prime}(t)\right| d t+\int_{E_{2}}\left|x^{\prime}(t)\right| d t \leq \tau_{1} c_{0}+\int_{E_{1}}\left|x^{\prime}(t)\right| d t \leq \\
\tau_{1} c_{0}+\int_{E_{1}} f\left(t, x(t), x^{\prime}(t)\right) d t \leq \tau_{1} c_{0}+I^{f}\left(T_{1}, T_{2}, x\right)+a \tau_{1} \leq \tau_{1}\left(c_{0}+a\right)+M_{1} .
\end{gathered}
$$

By this relation and $(2.20),(2.21) M_{2}-c_{0} \leq \tau_{1}\left(c_{0}+a\right)+M_{1}$. This is contradictory to (2.19). The obtained contradiction proves the proposition. 
Proposition 2.4. Let $M_{1}, \epsilon>0,0<\tau_{0}<\tau_{1}$. Then there exists a number $\delta>0$ such that for each $f \in \mathfrak{M}$, each numbers $T_{1}, T_{2}$ satisfying (2.14), each a.c. function $x:\left[T_{1}, T_{2}\right] \rightarrow K$ satisfying (2.15) and each $t_{1}, t_{2} \in\left[T_{1}, T_{2}\right]$ which satisfy $\left|t_{1}-t_{2}\right| \leq \delta$ the relation $\left|x\left(t_{1}\right)-x\left(t_{2}\right)\right| \leq \epsilon$ holds.

Proof. By Assumption (Aiii) and the properties of the function $\psi$ there exists a number $c_{0}>0$ such that

$$
f(t, x, u) \geq 4 \epsilon^{-1}\left(M_{1}+2+a \tau_{1}\right)|u|
$$

for each $f \in \mathfrak{M}$ and each $(t, x, u) \in[0, \infty) \times K \times R^{n}$ satisfying $|u| \geq c_{0}$. Fix a number

$$
\delta \in\left(0,8^{-1}\left(c_{0}+1\right)^{-1} \epsilon\right) .
$$

Assume that $f \in \mathfrak{M}$, numbers $T_{1}, T_{2}$ satisfy (2.14), an a. c. function $x:\left[T_{1}, T_{2}\right] \rightarrow K$ satisfies (2.15) and

$$
t_{1}, t_{2} \in\left[T_{1}, T_{2}\right], 0<\left|t_{1}-t_{2}\right| \leq \delta .
$$

Set

$$
E=\left[\inf \left\{t_{1}, t_{2}\right\}, \sup \left\{t_{1}, t_{2}\right\}\right], E_{1}=\left\{t \in E:\left|x^{\prime}(t)\right| \geq c_{0}\right\}, E_{2}=E \backslash E_{1} .
$$

It follows from (2.25), the definition of $c_{0},(2.23),(2.14)$ and Assumption (Aiii) that

$$
\begin{aligned}
\mid x\left(t_{2}\right)- & x\left(t_{1}\right)\left|\leq \int_{E_{1}}\right| x^{\prime}(t)\left|d t+\int_{E_{2}}\right| x^{\prime}(t)\left|d t \leq \delta c_{0}+\int_{E_{1}}\right| x^{\prime}(t) \mid d t \\
& \leq \delta c_{0}+\left[4\left(M_{1}+2+a \tau_{1}\right)\right]^{-1} \epsilon \int_{E_{1}} f\left(t, x(t), x^{\prime}(t)\right) d t \\
& \leq \delta c_{0}+\left[4\left(M_{1}+2+a \tau_{1}\right)\right]^{-1} \epsilon\left(I^{f}\left(T_{1}, T_{2}, x\right)+a \tau_{1}\right) .
\end{aligned}
$$

Together with $(2.15),(2.14)$ and $(2.24)$ this relation implies that

$$
\left|x\left(t_{2}\right)-x\left(t_{1}\right)\right| \leq \delta c_{0}+4^{-1} \epsilon \leq \epsilon .
$$

This completes the proof of the proposition.

We have the following result (see Berkovitz [4]).

Proposition 2.5. Assume that $f \in \mathfrak{M}, M_{1}>0,0 \leq T_{1}<T_{2}, x_{i}$ : $\left[T_{1}, T_{2}\right] \rightarrow K, i=1,2, \ldots$ is a sequence of a. c. functions such that

$$
I^{f}\left(T_{1}, T_{2}, x_{i}\right) \leq M_{1}, i=1,2, \ldots
$$

Then there exists a subsequence $\left\{x_{i_{k}}\right\}_{k=1}^{\infty}$ and an a. c. function $x:\left[T_{1}, T_{2}\right] \rightarrow$ $K$ such that

$$
\begin{gathered}
I^{f}\left(T_{1}, T_{2}, x\right) \leq M_{1}, x_{i_{k}} \rightarrow x(t) \text { as } k \rightarrow \infty \text { uniformly in }\left[T_{1}, T_{2}\right] \text { and } \\
x_{i_{k}}^{\prime} \rightarrow x^{\prime} \text { as } k \rightarrow \infty \text { weakly in } L^{1}\left(R^{n} ;\left(T_{1}, T_{2}\right)\right) .
\end{gathered}
$$


Corollary 2.1. For each $f \in \mathfrak{M}$, each pair of numbers $T_{1}, T_{2}$ satisfying $0 \leq$ $T_{1}<T_{2}$ and each $z_{1}, z_{2} \in K$ there exists an a.c. function $x:\left[T_{1}, T_{2}\right] \rightarrow K$ such that $x\left(T_{i}\right)=z_{i}, i=1,2, I^{f}\left(T_{1}, T_{2}, x\right)=U^{f}\left(T_{1}, T_{2}, z_{1}, z_{2}\right)$.

Corollary 2.2. For each $f \in \mathfrak{M}$, each $T_{1}, T_{2}$ satisfying $0 \leq T_{1}<T_{2}$ and each $z \in K$ there exists an a.c. function $x:\left[T_{1}, T_{2}\right] \rightarrow K$ such that $x\left(T_{1}\right)=$ $z, I^{f}\left(T_{1}, T_{2}, x\right)=\sigma^{f}\left(T_{1}, T_{2}, z\right)$.

It is an elementary exercise to prove the following result.

Proposition 2.6. Let $f \in \mathfrak{M}, 0<c_{1}<c_{2}<\infty$ and let $c_{3}>0$. Then there exists a neighborhood $U$ of $f$ in $\mathfrak{M}$ such that the set

$$
\begin{gathered}
\left\{U^{g}\left(T_{1}, T_{2}, z_{1}, z_{2}\right): g \in U, T_{1} \in[0, \infty), T_{2} \in\left[T_{1}+c_{1}, T_{1}+c_{2}\right],\right. \\
\left.z_{1}, z_{2} \in K,\left|z_{i}\right| \leq c_{3}, i=1,2\right\}
\end{gathered}
$$

is bounded.

Proposition 2.7. Assume that $K=R^{n}, f \in \mathfrak{M}, 0<c_{1}<c_{2}<\infty$ and $M, \epsilon>0$. Then there exists $\delta>0$ such that for each $T_{1}, T_{2} \geq 0$ satisfying

$$
T_{2}-T_{1} \in\left[c_{1}, c_{2}\right]
$$

and each $y_{1}, y_{2}, z_{1}, z_{2} \in R^{n}$ satisfying

$$
\left|y_{i}\right|,\left|z_{i}\right| \leq M, i=1,2, \quad \sup \left\{\left|y_{1}-y_{2}\right|,\left|z_{1}-z_{2}\right|\right\} \leq \delta
$$

the following relation holds:

$$
\left|U^{f}\left(T_{1}, T_{2}, y_{1}, z_{1}\right)-U^{f}\left(T_{1}, T_{2}, y_{2}, z_{2}\right)\right| \leq \epsilon
$$

Proof. By Proposition 2.6 there exists a number

$$
\begin{gathered}
M_{0}>\sup \left\{\left|U^{f}\left(T_{1}, T_{2}, y, z\right)\right|: T_{1} \in[0, \infty), T_{2} \in\left[T_{1}+c_{1}, T_{1}+c_{2}\right]\right. \\
\left.y, z \in R^{n},|y|,|z| \leq M\right\}
\end{gathered}
$$

By Proposition 2.3 there exists a number $M_{1}>0$ such that for each pair of numbers $T_{1}, T_{2} \geq 0$ satisfying (2.26) and each a.c. function $x:\left[T_{1}, T_{2}\right] \rightarrow R^{n}$ which satisfies $I^{f}\left(T_{1}, T_{2}, x\right) \leq 4 M_{0}+1$ the following relation holds:

$$
|x(t)| \leq M_{1}, t \in\left[T_{1}, T_{2}\right]
$$

Choose a number $\delta_{1}>0$ such that

$$
4 \delta_{1}\left(2 c_{2}+2 a+4 a c_{2}+1+M_{0}\right)<\epsilon
$$


(see Assumption (Aiii)). By Proposition 2.1 there exist

$$
\Gamma_{0}>2 \text { and } \delta_{2} \in\left(0,8^{-1}\right)
$$

such that

$$
\left|f\left(t, x_{1}, u_{1}\right)-f\left(t, x_{2}, u_{2}\right)\right| \leq \delta_{1} \inf \left\{f\left(t, x_{1}, u_{1}\right), f\left(t, x_{2}, u_{2}\right)\right\}
$$

for each $t \in[0, \infty)$ and each $u_{1}, u_{2}, x_{1}, x_{2} \in R^{n}$ which satisfy

$$
\left|x_{i}\right| \leq M_{1}+1,\left|u_{i}\right| \geq \Gamma_{0}-1, i=1,2,\left|u_{1}-u_{2}\right|,\left|x_{1}-x_{2}\right| \leq \delta_{2} .
$$

By Assumption (Aiv) there is a number

$$
\delta_{3} \in\left(0,4^{-1} \inf \left\{\delta_{1}, \delta_{2}\right\}\right)
$$

such that

$$
\left|f\left(t, x_{1}, u_{1}\right)-f\left(t, x_{2}, u_{2}\right)\right| \leq \delta_{1}
$$

for each $t \in[0, \infty)$, each $u_{1}, u_{2}, x_{1}, x_{2} \in R^{n}$ which satisfy

$$
\left|x_{i}\right|,\left|u_{i}\right| \leq \Gamma_{0}+M_{1}+4, i=1,2, \sup \left\{\left|x_{1}-x_{2}\right|,\left|u_{1}-u_{2}\right|\right\} \leq \delta_{3} .
$$

There exists a positive number $\delta$ such that

$$
8\left(c_{1}^{-1}+1\right) \delta<\delta_{3}
$$

Assume that numbers $T_{1}, T_{2} \geq 0$ satisfy (2.26) and $y_{1}, y_{2}, z_{1}, z_{2} \in R^{n}$ satisfy (2.27). By Corollary 2.1 there exists an a.c. function $x_{1}:\left[T_{1}, T_{2}\right] \rightarrow$ $R^{n}$ such that

$$
x_{1}\left(T_{1}\right)=y_{1}, x_{1}\left(T_{2}\right)=z_{1}, I^{f}\left(T_{1}, T_{2}, x_{1}\right)=U^{f}\left(T_{1}, T_{2}, y_{1}, z_{1}\right)
$$

Set

$x_{2}(t)=x_{1}(t)+y_{2}-y_{1}+\left(t-T_{1}\right)\left(T_{2}-T_{1}\right)^{-1}\left(z_{2}-z_{1}-y_{2}+y_{1}\right), t \in\left[T_{1}, T_{2}\right]$.

Clearly

$$
x_{2}\left(T_{1}\right)=y_{2}, x_{2}\left(T_{2}\right)=z_{2} .
$$

It follows from $(2.26),(2.27),(2.39),(2.29)$ and the definition of $M_{1}$ that

$$
\left|x_{1}(t)\right| \leq M_{1}, t \in\left[T_{1}, T_{2}\right]
$$


(2.40), (2.27) and (2.26) imply that

$$
\left|x_{1}(t)-x_{2}(t)\right| \leq 3 \delta,\left|x_{1}^{\prime}(t)-x_{2}^{\prime}(t)\right| \leq 2 c_{1}^{-1} \delta, t \in\left[T_{1}, T_{2}\right] .
$$

Set

$$
E_{1}=\left\{t \in\left[T_{1}, T_{2}\right]:\left|x_{1}^{\prime}(t)\right| \geq \Gamma_{0}\right\}, E_{2}=\left[T_{1}, T_{2}\right] \backslash E_{1} .
$$

We have

$$
\left|I^{f}\left(T_{1}, T_{2}, x_{2}\right)-I^{f}\left(T_{1}, T_{2}, x_{1}\right)\right| \leq \sigma_{1}+\sigma_{2}
$$

where

$$
\sigma_{j}=\int_{E_{j}}\left|f\left(t, x_{1}(t), x_{1}^{\prime}(t)\right)-f\left(t, x_{2}(t), x_{2}^{\prime}(t)\right)\right| d t, j=1,2 .
$$

We will estimate $\sigma_{1}, \sigma_{2}$ separately. Let $t \in E_{1}$. It follows from (2.42), (2.43), (2.44), (2.38), (2.35), (2.32) and the definition of $\delta_{2}$ that

$$
\left|f\left(t, x_{1}(t), x_{1}^{\prime}(t)\right)-f\left(t, x_{2}(t), x_{2}^{\prime}(t)\right)\right| \leq \delta_{1} f\left(t, x_{1}(t), x_{1}^{\prime}(t)\right) .
$$

Therefore $\sigma \leq \delta_{1} \int_{E_{1}} f\left(t, x_{1}(t), x_{1}^{\prime}(t)\right) d t$. This relation, Assumption (Aiii), (2.39), (2.27), (2.29) and (2.26) imply that

$$
\sigma_{1} \leq \delta_{1}\left(I^{f}\left(T_{1}, T_{2}, x_{1}\right)+a\left(T_{2}-T_{1}\right)\right) \leq \delta_{1}\left(M_{0}+a c_{2}\right) .
$$

Let $t \in E_{2}$. It follows from (2.42), (2.43), (2.38), (2.44) and the definition of $\delta_{3}$ that

$$
\left|f\left(t, x_{1}(t), x_{1}^{\prime}(t)\right)-f\left(t, x_{2}(t), x_{2}^{\prime}(t)\right)\right| \leq \delta_{1} .
$$

Therefore

$$
\sigma_{2} \leq \delta_{1} c_{2}
$$

Combining $(2.45),(2.47),(2.48)$ and $(2.31)$ we obtain that

$$
\left|I^{f}\left(T_{1}, T_{2}, x_{2}\right)-I^{f}\left(T_{1}, T_{2}, x_{1}\right)\right| \leq \delta_{1}\left(M_{0}+a c_{2}+c_{2}\right) \leq \epsilon .
$$

Together with (2.39) and (2.41) this implies that

$$
U^{f}\left(T_{1}, T_{2}, y_{2}, z_{2}\right) \leq U^{f}\left(T_{1}, T_{2}, y_{1}, z_{1}\right)+\epsilon .
$$

This completes the proof of the proposition. 
Proposition 2.8. Let $f \in \mathfrak{M}, 0<c_{1}<c_{2}<\infty, D, \epsilon>0$. Then there exists a neighborhood $V$ of $f$ in $\mathfrak{M}$ such that for each $g \in V$, each pair of numbers $T_{1}, T_{2} \geq 0$ satisfying $T_{2}-T_{1} \in\left[c_{1}, c_{2}\right]$ and each a. c. function $x:\left[T_{1}, T_{2}\right] \rightarrow K$ satisfying

$$
\inf \left\{I^{f}\left(T_{1}, T_{2}, x\right), I^{g}\left(T_{1}, T_{2}, x\right)\right\} \leq D
$$

the relation $\left|I^{f}\left(T_{1}, T_{2}, x\right)-I^{g}\left(T_{1}, T_{2}, x\right)\right| \leq \epsilon$ holds.

Proof. By Proposition 2.3 there exists a number $S>0$ such that for each $g \in \mathfrak{M}$, each $T_{1}, T_{2} \geq 0$ satisfying $T_{2}-T_{1} \in\left[c_{1}, c_{2}\right]$ and each a.c. function $x:\left[T_{1}, T_{2}\right] \rightarrow K$ which satisfies $I^{g}\left(T_{1}, T_{2}, x\right) \leq D+1$ the following relation holds:

$$
|x(t)| \leq S, t \in\left[T_{1}, T_{2}\right] .
$$

There exist $\delta \in(0,1), N>S$ and $\Gamma>1$ such that $(2.51) \delta\left(c_{2}+1\right) \leq 4^{-1} \epsilon, \psi(N) N>4 a,(\Gamma-1)\left(c_{2}+D+a c_{2}+1\right) \leq 4^{-1} \epsilon$.

Set $V=\{g \in \mathfrak{M}:(f, g) \in E(N, \delta, \Gamma)\}$ (see (1.1)). Assume that $g \in V$,

$$
T_{1}, T_{2} \geq 0, T_{2}-T_{1} \in\left[c_{1}, c_{2}\right]
$$

and $x:\left[T_{1}, T_{2}\right] \rightarrow K$ is an a.c. function satisfying (2.49). It follows from the definition of $S$ that (2.50) holds. Set

$$
E_{1}=\left\{t \in\left[T_{1}, T_{2}\right]:\left|x^{\prime}(t)\right| \leq N\right\}, E_{2}=\left[T_{1}, T_{2}\right] \backslash E_{1} .
$$

It follows from (2.50) and the definition of $V$ and $N$ that

$$
\left|f\left(t, x(t), x^{\prime}(t)\right)-g\left(t, x(t), x^{\prime}(t)\right)\right| \leq \delta, t \in E_{1} .
$$

Define

$$
h(t)=\inf \left\{f\left(t, x(t), x^{\prime}(t)\right), g\left(t, x(t), x^{\prime}(t)\right)\right\}, t \in\left[T_{1}, T_{2}\right] .
$$

It follows from (2.50), (2.51), Assumption (Aiii) and the definition of $V, N$ that for $t \in E_{2}$

$$
\begin{gathered}
\left(f\left(t, x(t), x^{\prime}(t)\right)+1\right)\left(g\left(t, x(t), x^{\prime}(t)\right)+1\right)^{-1} \in\left[\Gamma^{-1}, \Gamma\right], \\
\left|f\left(t, x(t), x^{\prime}(t)\right)-g\left(t, x(t), x^{\prime}(t)\right)\right| \leq(\Gamma-1)(h(t)+1) .
\end{gathered}
$$

By (2.53), (2.52), (2.55), (2.49), (2.54), Assumption (Aiii) and (2.51)

$$
\begin{gathered}
\left|I^{f}\left(T_{1}, T_{2}, x\right)-I^{g}\left(T_{1}, T_{2}, x\right)\right| \leq \int_{E_{1}}\left|f\left(t, x(t), x^{\prime}(t)\right)-g\left(t, x(t), x^{\prime}(t)\right)\right| d t \\
+\int_{E_{2}}\left|f\left(t, x(t), x^{\prime}(t)\right)-g\left(t, x(t), x^{\prime}(t)\right)\right| d t \leq \delta c_{2}+(\Gamma-1) \int_{E_{2}}(h(t)+1) d t \\
\leq \delta c_{2}+(\Gamma-1) c_{2}+(\Gamma-1)\left(D+a c_{2}\right) \leq \epsilon .
\end{gathered}
$$

The proposition is proved. 
Proposition 2.9. Let $f \in \mathfrak{M}, 0<c_{1}<c_{2}<\infty, c_{3}, \epsilon>0$. Then there exists a neighborhood $V$ of $f$ in $\mathfrak{M}$ such that for each $g \in V$, each $T_{1}, T_{2} \geq 0$ satisfying $T_{2}-T_{1} \in\left[c_{1}, c_{2}\right]$ and each $y, z \in K$ satisfying $|y|,|z| \leq c_{3}$ the relation

$$
\left|U^{f}\left(T_{1}, T_{2}, y, z\right)-U^{g}\left(T_{1}, T_{2}, y, z\right)\right| \leq \epsilon
$$

holds.

Proof. By Proposition 2.6 there exist a neighborhood $V_{1}$ of $f$ in $\mathfrak{M}$ and a number

$$
\begin{gathered}
D_{0}>\sup \left\{\left|U^{g}\left(T_{1}, T_{2}, z_{1}, z_{2}\right)\right|: g \in V_{1}, T_{1} \in[0, \infty), T_{2} \in\left[T_{1}+c_{1}, T_{1}+c_{2}\right],\right. \\
\left.z_{1}, z_{2} \in K,\left|z_{i}\right| \leq c_{3}, i=1,2\right\} .
\end{gathered}
$$

By Proposition 2.8 there exists a neighborhood $V$ of $f$ in $\mathfrak{M}$ such that $V \subset V_{1}$ and for each $g \in V$, each $T_{1}, T_{2} \geq 0$ satisfying $T_{2}-T_{1} \in\left[c_{1}, c_{2}\right]$ and each a.c. function $x:\left[T_{1}, T_{2}\right] \rightarrow K$ satisfying

$$
\inf \left\{I^{f}\left(T_{1}, T_{2}, x\right), I^{g}\left(T_{1}, T_{2}, x\right)\right\} \leq D_{0}+2
$$

the relation $\left|I^{f}\left(T_{1}, T_{2}, x\right)-I^{g}\left(T_{1}, T_{2}, x\right)\right| \leq \inf \{1, \epsilon\}$ holds.

To complete the proof it remains now to note that for $g \in V, T_{1} \geq 0$, $T_{2} \in\left[T_{1}+c_{1}, T_{1}+c_{2}\right]$ and $y, z \in K$ satisfying $|y|,|z| \leq c_{3}$ the following relation holds:

$$
\begin{gathered}
U^{g}\left(T_{1}, T_{2}, y, z\right)=\inf \left\{I^{g}\left(T_{1}, T_{2}, x\right): x:\left[T_{1}, T_{2}\right] \rightarrow K\right. \text { is an a.c. function } \\
\text { satisfying } \left.x\left(T_{1}\right)=y, x\left(T_{2}\right)=z, I^{g}\left(T_{1}, T_{2}, x\right) \leq D_{0}+1\right\} .
\end{gathered}
$$

\section{Discrete-time CONTRol Systems}

Let $f \in \mathfrak{M}, \bar{z} \in K$ and let $0<c_{1}<c_{2}<\infty$. By Proposition 2.6 there exists a neighborhood $U_{0}$ of $f$ in $\mathfrak{M}$ and a number

$$
\begin{gathered}
M_{0} \geq \sup \left\{\left|U^{g}\left(T_{1}, T_{2}, y, z\right)\right|: g \in U_{0}, T_{1} \in[0, \infty), T_{2} \in\left[T_{1}+c_{1}, T_{1}+c_{2}\right],\right. \\
y, z \in K,|y|,|z| \leq 2|\bar{z}|+1\} .
\end{gathered}
$$

By Proposition 2.3 there exists a positive number $M_{1}$ such that

$$
\begin{gathered}
\inf \left\{U^{g}\left(T_{1}, T_{2}, y, z\right): g \in \mathfrak{M}, T_{1} \in[0, \infty), T_{2} \in\left[T_{1}+c_{1}, T_{1}+c_{2}\right]\right. \\
\left.y, z \in K,|y|+|z| \geq M_{1}\right\}>2 M_{0}+1 .
\end{gathered}
$$


Proposition 3.1. Assume that a positive number $M_{1}$ satisfies (3.2) and $M_{2}>0$. Then there exists a neighborhood $U$ of $f$ in $\mathfrak{M}$ and an integer $N>2$ such that:

1. For each $g \in U$, each $\Delta \in[0, \infty)$, each $T \in\left[c_{1}, c_{2}\right]$, each pair of integers $q_{1}, q_{2}$ satisfying $0 \leq q_{1}<q_{2}, q_{2}-q_{1} \geq N$ and each sequence $\left\{z_{i}\right\}_{i=q_{1}}^{q_{2}} \subset K$ satisfying

$$
\left\{i \in\left\{q_{1}, \ldots q_{2}\right\}:\left|z_{i}\right| \leq M_{1}\right\}=\left\{q_{1}, q_{2}\right\}
$$

the following relation holds:

$\sum_{i=q_{1}}^{q_{2}-1}\left[U^{g}\left(\Delta+i T, \Delta+(i+1) T, z_{i}, z_{i+1}\right)-U^{g}\left(\Delta+i T, \Delta+(i+1) T, y_{i}, y_{i+1}\right)\right] \geq M_{2}$ where $y_{i}=z_{i}, i=q_{1}, q_{2}, y_{i}=\bar{z}, i=q_{1}+1, \ldots q_{2}-1$;

2. For each $g \in U$, each $\Delta \in[0, \infty)$, each $T \in\left[c_{1}, c_{2}\right]$, each pair of integers $q_{1}, q_{2}$ satisfying $0 \leq q_{1}<q_{2}, q_{2}-q_{1} \geq N$ and each sequence $\left\{z_{i}\right\}_{i=q_{1}}^{q_{2}} \subset K$ satisfying

$$
\left\{i \in\left\{q_{1}, \ldots, q_{2}\right\}:\left|z_{i}\right| \leq M_{1}\right\}=\left\{q_{1}\right\}
$$

relation (3.3) holds with $y_{q_{1}}=z_{q_{1}}, y_{i}=\bar{z}, i=q_{1}+1, \ldots, q_{2}$.

Proof. By Proposition 2.6 there exists a neighborhood $U$ of $f$ in $\mathfrak{M}$ and a number $M_{3}>0$ such that

$$
\begin{gathered}
U \subset U_{0}, M_{3} \geq \sup \left\{\left|U^{g}\left(T_{1}, T_{2}, y, z\right)\right|: g \in U, T_{1} \in[0, \infty),\right. \\
\left.T_{2} \in\left[T_{1}+c_{1}, T_{1}+c_{2}\right], y, z \in K,|y|,|z| \leq 2|\bar{z}|+1+2 M_{1}\right\} .
\end{gathered}
$$

Fix an integer $N \geq M_{2}+4 M_{3}+4$. The validity of the proposition now follows from the definition of $U, M_{3}, N$ and (3.1), (3.2).

Proposition 3.2. Assume that a positive number $M_{1}$ satisfies (3.2) and $M_{3}>0$. Then there exists a neighborhood $V$ of $f$ in $\mathfrak{M}$ and a number $M_{4}>M_{1}$ such that:

1. For each $g \in V$, each $\Delta \in[0, \infty)$, each $T \in\left[c_{1}, c_{2}\right]$, each pair of integers $q_{1}, q_{2}$ satisfying $0 \leq q_{1}<q_{2}$ and each sequence $\left\{z_{i}\right\}_{i=q_{1}}^{q_{2}} \subset K$ satisfying

$$
\sup \left\{\left|z_{q_{1}}\right|,\left|z_{q_{2}}\right|\right\} \leq M_{1}, \sup \left\{\left|z_{i}\right|: i=q_{1}, \ldots q_{2}\right\}>M_{4}
$$

there is a sequence $\left\{y_{i}\right\}_{i=q_{1}}^{q_{2}} \subset K$ which satisfies $y_{q_{j}}=z_{q_{j}}, j=1,2$,

$\sum_{i=q_{1}}^{q_{2}-1}\left[U^{g}\left(\Delta+i T, \Delta+(i+1) T, z_{i}, z_{i+1}\right)-U^{g}\left(\Delta+i T, \Delta+(i+1) T, y_{i}, y_{i+1}\right)\right] \geq M_{3}$.

2. For each $g \in V$, each $\Delta \in[0, \infty)$, each $T \in\left[c_{1}, c_{2}\right]$, each pair of integers $q_{1}, q_{2}$ satisfying $0 \leq q_{1}<q_{2}$ and each sequence $\left\{z_{i}\right\}_{i=q_{1}}^{q_{2}} \subset K$ satisfying

$$
\left|z_{q_{1}}\right| \leq M_{1}, \sup \left\{\left|z_{i}\right|: i=q_{1}, \ldots q_{2}\right\}>M_{4}
$$


there is a sequence $\left\{y_{i}\right\}_{i=q_{1}}^{q_{2}} \subset K$ which satisfies $y_{q_{1}}=z_{q_{1}}$ and (3.5).

Proof. There exist a neighborhood $U$ of $f$ in $\mathfrak{M}$ and an integer $N>2$ such that Proposition 3.1 holds with $M_{2}=4\left(M_{3}+1\right)$ and $U \subset U_{0}$. By Proposition 2.6 there exist a neighborhood $V$ of $f$ in $\mathfrak{M}$ and a number $r_{1}$ such that

$$
\begin{gathered}
V \subset U, r_{1}>\sup \left\{\left|U^{g}\left(T_{1}, T_{2}, y, z\right)\right|: g \in V, T_{1} \in[0, \infty), T_{2} \in\right. \\
\left.\left[T_{1}+c_{1}, T_{1}+c_{2}\right], y, z \in K,|y|,|z| \leq|\bar{z}|+1+M_{1}\right\} .
\end{gathered}
$$

By Proposition 2.3 there exists a positive number $M_{4}>M_{1}$ such that

$$
\begin{gathered}
\inf \left\{U^{g}\left(T_{1}, T_{2}, y, z\right): g \in \mathfrak{M}, T_{1} \in[0, \infty), T_{2} \in\left[T_{1}+c_{1}, T_{1}+c_{2}\right],\right. \\
\left.y, z \in K,|y|+|z| \geq M_{4}\right\}>3 r_{1} N+4+4 M_{3}+3 a c_{2} N
\end{gathered}
$$

(recall $a$ in Assumption (Aiii)).

We will prove Assertion 1. Let $g \in V, \Delta \in[0, \infty), T \in\left[c_{1}, c_{2}\right], 0 \leq q_{1}<$ $q_{2},\left\{z_{i}\right\}_{i=q_{1}}^{q_{2}} \subset K$. Assume that (3.4) holds. Then there is $j \in\left\{q_{1}, \ldots q_{2}\right\}$ such that $\left|z_{j}\right|>M_{4}$. Set

$$
i_{1}=\sup \left\{i \in\left\{q_{1}, \ldots, j\right\}:\left|z_{i}\right| \leq M_{1}\right\}, i_{2}=\inf \left\{i \in\left\{j, \ldots q_{2}\right\}:\left|z_{i}\right| \leq M_{1}\right\}
$$

If $i_{2}-i_{1} \geq N$ then by the definition of $V, U, N$ and Proposition 3.1 there exists a sequence $\left\{y_{i}\right\}_{i=q_{1}}^{q_{2}} \subset K$ which satisfies (3.5) and $y_{q_{i}}=z_{q_{i}}, i=1,2$.

Assume that $i_{2}-i_{1}<N$ and define a sequence $\left\{y_{i}\right\}_{i=q_{1}}^{q_{2}} \subset K$ by

$$
y_{i}=z_{i}, i \in\left\{q_{1}, \ldots i_{1}\right\} \cup\left\{i_{2}, \ldots q_{2}\right\}, y_{i}=\bar{z}, i=i_{1}+1 \ldots i_{2}-1
$$

It follows from (3.9), (3.7), Assumption (Aiii) and the definition of $i_{1}, i_{2}, j$ that

$$
\begin{aligned}
& \sum_{i=q_{1}}^{q_{2}-1}\left[U^{g}\left(\Delta+i T, \Delta+(i+1) T, z_{i}, z_{i+1}\right)-U^{g}\left(\Delta+i T, \Delta+(i+1) T, y_{i}, y_{i+1}\right)\right] \\
& =\sum_{i=i_{1}}^{i_{2}-1}\left[U^{g}\left(\Delta+i T, \Delta+(i+1) T, z_{i}, z_{i+1}\right)-U^{g}\left(\Delta+i T, \Delta+(i+1) T, y_{i}, y_{i+1}\right)\right] \\
& \quad \geq U^{g}\left(\Delta+(j-1) T, \Delta+j T, z_{j-1}, z_{j}\right)-a\left(i_{2}-i_{1}-1\right) c_{2}-\left(i_{2}-i_{1}\right) r_{1} .
\end{aligned}
$$

By this relation and the definition of $j, M_{4}$ (see (3.8))

$$
\sum_{i=q_{1}}^{q_{2}-1}\left[U^{g}\left(\Delta+i T, \Delta+(i+1) T, z_{i}, z_{i+1}\right)-\right.
$$




$$
\left.U^{g}\left(\Delta+i T, \Delta+(i+1) T, y_{i}, y_{i+1}\right)\right] \geq 4 M_{3}+4 .
$$

This completes the proof of Assertion 1.

We will prove Assertion 2. Let $g \in V, \Delta \in[0, \infty), T \in\left[c_{1}, c_{2}\right], 0 \leq q_{1}<$ $q_{2},\left\{z_{i}\right\}_{i=q_{1}}^{q_{2}} \subset K$. Assume that (3.6) holds. Then there is $j \in\left\{q_{1}, \ldots q_{2}\right\}$ such that $\left|z_{j}\right|>M_{4}$. Set $i_{1}=\sup \left\{i \in\left\{q_{1}, \ldots, j\right\}:\left|z_{i}\right| \leq M_{1}\right\}$.

There are two cases: 1) $\left.\left|z_{i}\right|>M_{1}, i=j, \ldots, q_{2}, ; 2\right) \inf \left\{\left|z_{i}\right|: i=\right.$ $\left.j, \ldots q_{2}\right\} \leq M_{1}$. Consider the first case. We set

$$
y_{i}=z_{i}, i=q_{1}, \ldots i_{1}, y_{i}=\bar{z}, i=i_{1}+1, \ldots q_{2} \text {. }
$$

If $q_{2}-i_{1} \geq N$ then (3.5) follows from the definition of $V, U, N$ and Proposition 3.1. If $q_{2}-i_{1}<N$ then (3.5) follows from the definition of $\left\{y_{i}\right\}_{i=q_{1}}^{q_{2}}$, $i_{1}, j, M_{4},(3.7)$ (see $(3.10)$, (3.11) with $\left.i_{2}=q_{2}\right)$.

Consider the second case. Set $i_{2}=\inf \left\{i \in\left\{j, \ldots q_{2}\right\}:\left|z_{i}\right| \leq M_{1}\right\}$. If $i_{2}-i_{1} \geq N$ then by the definition of $V, U, N$ and Proposition 3.1 there exists a sequence $\left\{y_{i}\right\}_{i=q_{1}}^{q_{2}} \subset K$ which satisfies (3.5) and $y_{q_{i}}=z_{q_{i}}, i=1,2$. If $i_{2}-i_{1}<N$ we define a sequence $\left\{y_{i}\right\}_{i=q_{1}}^{q_{2}} \subset K$ by (3.9). Then (3.10) and (3.11) follows from (3.9), the definition of $i_{1}, i_{2}, j, M_{4},(3.7)$. Assertion 2 is proved. This completes the proof of the proposition.

\section{Proof of Theorems 1.1-1.3}

Construction of a neighborhood $U$. Let $f \in \mathfrak{M}, \bar{z} \in K, M>2|\bar{z}|$. By Proposition 2.6 there exist a neighborhood $U_{0}$ of $f$ in $\mathfrak{M}$ and a number

$$
\begin{gathered}
M_{0} \geq \sup \left\{\left|U^{g}\left(T_{1}, T_{2}, y, z\right)\right|: g \in U_{0}, T_{1} \in[0, \infty),\right. \\
\left.T_{2} \in\left[T_{1}+4^{-1}, T_{1}+4\right], y, z \in K,|y|,|z| \leq 2|\bar{z}|+1\right\} .
\end{gathered}
$$

By Proposition 2.3 there exists a number $M_{1}>M$ such that

$$
\begin{gathered}
\inf \left\{U^{g}\left(T_{1}, T_{2}, y, z\right): g \in \mathfrak{M}, T_{1} \in[0, \infty), T_{2} \in\left[T_{1}+4^{-1}, T_{1}+4\right]\right. \\
\left.y, z \in K,|y|+|z| \geq M_{1}\right\}>2 M_{0}+1
\end{gathered}
$$

By (4.1), (4.2) there exists a neighborhood $U_{1}$ of $f$ in $\mathfrak{M}$ and a number $M_{2}$ such that

$$
\begin{gathered}
U_{1} \subset U_{0}, M_{2}>M_{1} \text { and Proposition } 3.2 \text { holds with } M_{3}=1, \\
c_{1}=4^{-1}, c_{2}=4, V=U_{1}, M_{4}=M_{2} .
\end{gathered}
$$

By Proposition 2.6 there exist a neighborhood $U_{2}$ of $f$ in $\mathfrak{M}$ and a number $Q_{0}>0$ such that

$$
U_{2} \subset U_{1}, Q_{0}>\sup \left\{\left|U^{g}\left(T_{1}, T_{2}, y, z\right)\right|: g \in U_{2}, T_{1} \in[0, \infty)\right.
$$




$$
\left.T_{2} \in\left[T_{1}+4^{-1}, T_{1}+4\right], y, z \in K,|y|,|z| \leq M_{2}+1\right\} .
$$

By Proposition 2.3 there exists a number

$$
Q_{1}>Q_{0}+M_{2}+1
$$

such that

$$
|x(t)| \leq Q_{1}, t \in\left[T_{1}, T_{2}\right]
$$

for each $g \in \mathfrak{M}$, each $T_{1}, T_{2}$ satisfying

$$
0 \leq T_{1}<T_{2}, T_{2}-T_{1} \in\left[4^{-1}, 4\right]
$$

and each a.c. function $x:\left[T_{1}, T_{2}\right] \rightarrow K$ which satisfies $I^{g}\left(T_{1}, T_{2}, x\right) \leq$ $2 Q_{0}+2$.

By Proposition 2.6 there exist a neighborhood $U$ of $f$ in $\mathfrak{M}$ and a number $Q_{2}>0$ such that

$$
\begin{gathered}
U \subset U_{2}, Q_{2}>Q_{1}, Q_{2}>\sup \left\{\left|U^{g}\left(T_{1}, T_{2}, y, z\right)\right|: g \in U,\right. \\
\left.T_{1} \in[0, \infty), T_{2} \in\left[T_{1}+4^{-1}, T_{1}+4\right], y, z \in K,|y|,|z| \leq 2 Q_{1}+4\right\} .
\end{gathered}
$$

We may assume without loss of generality that there exists a number

$$
\begin{gathered}
Q_{3}>\sup \left\{|g(t, y, u)|: g \in U, t \in[0, \infty), y \in K, u \in R^{n},\right. \\
\left.|y|,|u| \leq 2 M_{2}+2\right\} .
\end{gathered}
$$

Construction of a function $Z^{g}:[0, \infty) \rightarrow K$. Let $g \in U, z \in K,|z| \leq M$. By Corollary 2.2 for any integer $q \geq 1$ there exists an a. c. function $Z_{q}^{g}:[0, q] \rightarrow K$ such that

$$
Z_{q}^{g}(0)=z, I^{g}\left(0, q, Z_{q}^{g}\right)=\sigma^{g}(0, q, z) .
$$

It follows from Proposition 3.2 and the definition of $Z_{q}^{g}, U_{1}, M_{2}$ that

$$
\left|Z_{q}^{g}(i)\right| \leq M_{2}, i=0, \ldots q, q=1,2, \ldots .
$$

There exists a subsequence $\left\{Z_{g_{j}}^{g}\right\}_{j=1}^{\infty}$ such that for any integer $i \geq 0$ there exists

$$
z_{i}^{g}=\lim _{j \rightarrow \infty} Z_{q_{j}}^{g}(i)
$$

By Corollary 2.1 there exists an a.c. fnction $Z^{g}:[0, \infty) \rightarrow K$ such that for each integer $i \geq 0$

$$
Z^{g}(i)=z_{i}^{g}, I^{g}\left(i, i+1, Z^{g}\right)=U^{g}\left(i, i+1, z_{i}^{g}, z_{i+1}^{g}\right) .
$$

It follows from (4.9), (4.10) and (4.4) that

$$
I^{g}\left(i, i+1, Z_{q}^{g}\right)<Q_{0}, i=0, \ldots q-1, q=1,2, \ldots
$$

(4.10), (4.11), (4.12) and (4.4) imply that

$$
I^{g}\left(i, i+1, Z^{g}\right)<Q_{0}, i=0,1, \ldots
$$

By (4.13), (4.14) and the definition of $Q_{1}$ (see (4.5), (4.6))

$$
\left|Z_{q}^{g}(t)\right| \leq Q_{1}, t \in[0, q], q=1,2, \ldots, \quad\left|Z^{g}(t)\right| \leq Q_{1}, t \in[0, \infty) .
$$

Therefore for each $g \in U$ and each $z \in K$ satisfying $|z| \leq M$ we define a.c. functions $Z_{q}^{g}:[0, q] \rightarrow K, q=1,2, \ldots$ and $Z^{g}:[0, \infty) \rightarrow K$ satisfying (4.9)-(4.15). 
Lemma 4.1. Let $g \in U, z \in K,|z| \leq M$. Then for each pair of integers $q_{1}, q_{2}$ satisfying $0 \leq q_{1}<q_{2}$ and each sequence $\left\{y_{i}\right\}_{i=q_{1}}^{q_{2}} \subset K$ satisfying $\left|y_{q_{1}}\right| \leq M_{1}$ the following relation holds:

$$
\sum_{i=q_{1}}^{q_{2}-1}\left[U^{g}\left(i, i+1, z_{i}^{g}, z_{i+1}^{g}\right)-U^{g}\left(i, i+1, y_{i}, y_{i+1}\right)\right] \leq 4+4 Q_{2} .
$$

Proof. Assume that integers $q_{1}, q_{2}$ satisfy $0 \leq q_{1}<q_{2}$ and a sequence $\left\{y_{i}\right\}_{i=q_{1}}^{q_{2}} \subset K$ satisfies $\left|y_{q_{1}}\right| \leq M_{1}$. We will show that (4.16) holds.

Let us assume the converse. Then

$$
\sum_{i=q_{1}}^{q_{2}-1}\left[U^{g}\left(i, i+1, z_{i}^{g}, z_{i+1}^{g}\right)-U^{g}\left(i, i+1, y_{i}, y_{i+1}\right)\right]>4+4 Q_{2} .
$$

By Corollaries 2.1, 2.2 we may assume without loss of generality that

$$
\sum_{i=q_{1}}^{q_{2}-1}\left[U^{g}\left(i, i+1, y_{i}, y_{i+1}\right)-U^{g}\left(i, i+1, \bar{y}_{i}, \bar{y}_{i+1}\right)\right] \leq 0
$$

for each sequence $\left\{\bar{y}_{i}\right\}_{i=q_{1}}^{q_{2}} \subset K$ satisfying $\bar{y}_{q_{1}}=y_{q_{1}}$. It follows from (4.3), (4.5) that

$$
\left|y_{i}\right| \leq M_{2}<Q_{1}, i=q_{1}, \ldots q_{2}
$$

By Proposition 2.5, (4.9), (4.11) and (4.13) for any integer $i \geq 0$

$$
U^{g}\left(i, i+1, z_{i}^{g}, z_{i+1}^{g}\right) \leq \liminf _{j \rightarrow \infty} U^{g}\left(i, i+1, Z_{q_{j}}^{g}(i), Z_{q_{j}}^{g}(i+1)\right)
$$

Therefore there exists an integer $q>q_{2}+1$ such that

$$
\sum_{i=q_{1}}^{q_{2}}\left[U^{g}\left(i, i+1, z_{i}^{g}, z_{i+1}^{g}\right)-U^{g}\left(i, i+1, Z_{q}^{g}(i), Z_{q}^{g}(i+1)\right)\right] \leq 1 .
$$

We define a sequence $\left\{h_{i}\right\}_{i=0}^{q} \subset K$ as follows

(4.20) $h_{i}=Z_{q}^{g}(i), i \in\left\{0, \ldots q_{1}\right\} \cup\left\{q_{2}+1, \ldots q\right\}, h_{i}=y_{i}, i=q_{1}+1, \ldots q_{2}$.

It follows from (4.20), (4.9), Corollary 2.1, (4.19) and (4.17) that

$$
0 \geq \sum_{i=0}^{q-1}\left[U^{g}\left(i, i+1, Z_{q}^{g}(i), Z_{q}^{g}(i+1)\right)-U^{g}\left(i, i+1, h_{i}, h_{i+1}\right)\right]
$$




$$
\begin{gathered}
=\sum_{i=q_{1}}^{q_{2}}\left[U^{g}\left(i, i+1, Z_{q}^{g}(i), Z_{q}^{g}(i+1)\right)-U^{g}\left(i, i+1, h_{i}, h_{i+1}\right)\right] \\
=\sum_{i=q_{1}}^{q_{2}}\left[U^{g}\left(i, i+1, Z_{q}^{g}(i), Z_{q}^{g}(i+1)\right)-U^{g}\left(i, i+1, z_{i}^{g}, z_{i+1}^{g}\right)\right] \\
+\sum_{i=q_{1}}^{q_{2}} U^{g}\left(i, i+1, z_{i}^{g}, z_{i+1}^{g}\right)-\sum_{i=q_{1}}^{q_{2}-1} U^{g}\left(i, i+1, y_{i}, y_{i+1}\right)+U^{g}\left(q_{1}, q_{1}+1, y_{q_{1}}, y_{q_{1}+1}\right) \\
-U^{g}\left(q_{1}, q_{1}+1, h_{q_{1}}, h_{q_{1}+1}\right)-U^{g}\left(q_{2}, q_{2}+1, h_{q_{2}}, h_{q_{2}+1}\right) \geq 3+4 Q_{2} \\
+U^{g}\left(q_{2}, q_{2}+1,, z_{q_{2}}^{g}, z_{q_{2}+1}^{g}\right)+U^{g}\left(q_{1}, q_{1}+1, y_{q_{1}}, y_{q_{1}+1}\right) \\
-U^{g}\left(q_{1}, q_{1}+1, h_{q_{1}}, h_{q_{1}+1}\right)-U^{g}\left(q_{2}, q_{2}+1, h_{q_{2}}, h_{q_{2}+1}\right) .
\end{gathered}
$$

Together with (4.20), (4.18), (4.10), (4.11), (4.5) and (4.7) this relation implies that

$$
0 \geq 3+4 Q+U^{g}\left(q_{2}, q_{2}+1, z_{q_{2}}^{g}, z_{q_{2}+1}^{g}\right)+U^{g}\left(q_{1}, q_{1}+1, y_{q_{1}}, y_{q_{1}+1}\right)
$$

$-U^{g}\left(q_{1}, q_{1}+1, Z_{q}^{g}\left(q_{1}\right), y_{q_{1}+1}\right)-U^{g}\left(q_{2}, q_{2}+1, y_{q_{2}}, Z_{q}^{g}\left(q_{2}+1\right)\right) \geq 3+4 Q_{2}-4 Q_{2}$.

The obtained contradiction proves the lemma.

Lemma 4.2. Let $g \in U, z \in K,|z| \leq M$, an integer $q \geq 0, T \in(q, \infty)$ and let $x:[q, T] \rightarrow K$ be an a.c. function satisfying $|x(q)| \leq M_{1}$. Then

$$
I^{g}\left(q, T, Z^{g}\right) \leq I^{g}(q, T, x)+4+4 Q_{2}+Q_{0}+2 a
$$

(recall a in Assumption (Aiii)).

Proof. There exists an integer $q_{1} \geq q$ such that $q_{1}<T \leq q_{1}+1$. It follows from Lemma 4.1 and (4.12) that

$$
I^{g}\left(q, q_{1}, Z^{g}\right) \leq I^{g}\left(q, q_{1}, x\right)+4+4 Q_{2} .
$$

By Assumption (Aiii) and (4.14)

$$
I^{g}\left(q_{1}, T, x\right) \geq-a, \quad I^{g}\left(q_{1}, T, Z^{g}\right) \leq Q_{0}+a .
$$

(4.22) and (4.23) imply (4.21). The lemma is proved. 
Lemma 4.3. Let $g \in U, z \in K,|z| \leq M, 0 \leq T_{1}<T_{2}$ and let $x:\left[T_{1}, T_{2}\right] \rightarrow$ $K$ be an a.c. function satisfying $\left|x\left(T_{1}\right)\right| \leq M_{1}$. Then

$$
I^{g}\left(T_{1}, T_{2}, Z^{g}\right) \leq I^{g}\left(T_{1}, T_{2}, x\right)+4+4 Q_{2}+Q_{0}+Q_{3}+3 a .
$$

Proof. There exists an integer $q \geq 0$ such that $q \leq T_{1}<q+1$. Set

$$
x_{1}(t)=x\left(T_{1}\right), t \in\left[q, T_{1}\right], x_{1}(t)=x(t), t \in\left[T_{1}, T_{2}\right] .
$$

By Lemma 4.2

$$
I^{g}\left(q, T_{2}, Z^{g}\right) \leq I^{g}\left(q, T_{2}, x_{1}\right)+4+4 Q_{2}+Q_{0}+2 a .
$$

By Assumption (Aiii) and (4.26)

$$
\begin{gathered}
I^{g}\left(T_{1}, T_{2}, Z^{g}\right)=I^{g}\left(q, T_{2}, Z^{g}\right)-I^{g}\left(q, T_{1}, Z^{g}\right) \leq I^{g}\left(q, T_{2}, Z^{g}\right)+a \leq \\
I^{g}\left(q, T_{2}, x_{1}\right)+4+4 Q_{2}+Q_{0}+3 a .
\end{gathered}
$$

It follows from (4.25) and (4.8) that $\left|I^{g}\left(q, T_{1}, x_{1}\right)\right| \leq Q_{3}$. (4.24) now follows from this relation and (4.27), (4.25). The lemma is proved.

Lemma 4.4. Let $g \in U, z \in K,|z| \leq M,\left\{y_{i}\right\}_{i=0}^{\infty} \subset K$,

$$
\limsup _{i \rightarrow \infty}\left|y_{i}\right|>M_{2}
$$

Then

$$
\sum_{i=0}^{N-1}\left[U^{g}\left(i, i+1, y_{i}, y_{i+1}\right)-U^{g}\left(i, i+1, z_{i}^{g}, z_{i+1}^{g}\right)\right] \rightarrow \infty \text { as } N \rightarrow \infty
$$

Proof. There are two cases:
a) $\liminf _{i \rightarrow \infty}\left|y_{i}\right|>2^{-1} M_{1}$
b) $\liminf _{i \rightarrow \infty}\left|y_{i}\right| \leq 2^{-1} M_{1}$.

Consider the case a). Set $h_{i}=\bar{z}$ for $i=0,1, \ldots$ It follows from (4.1), (4.2) that

$$
U^{g}\left(i, i+1, y_{i}, y_{i+1}\right)-U^{g}\left(i, i+1, h_{i}, h_{i+1}\right) \geq M_{0}+1
$$

for all large $i$. (4.29) now follows from this relation and Lemma 4.1.

Consider the case b). By (4.28) there exists a subsequence $\left\{y_{i_{k}}\right\}_{k=1}^{\infty}$ such that

(4.30) $0<i_{1},\left|y_{i_{k}}\right|<M_{1}, \sup \left\{\left|y_{j}\right|: j=i_{k}, \ldots i_{k+1}\right\}>M_{2}, k=1,2, \ldots$ 
It follows from (4.3), (4.30) and Proposition 3.2 that for any integer $k \geq 1$ there exists a sequence $\left\{h_{j}\right\}_{j=i_{k}}^{i_{k+1}} \subset K$ such that $h_{j}=y_{j}, j \in\left\{i_{k}, i_{k+1}\right\}$,

$$
\sum_{j=i_{k}}^{i_{k+1}-1}\left[U^{g}\left(j, j+1, y_{j}, y_{j+1}\right)-U^{g}\left(j, j+1, h_{j}, h_{j+1}\right)\right] \geq 1 .
$$

Fix an integer $q \geq 4$. By (4.30), Lemma 4.1 and (4.31) for an integer $N>i_{q}$

$$
\begin{aligned}
\sum_{j=i_{q}}^{N-1}[ & \left.U^{g}\left(j, j+1, z_{j}^{g}, z_{j+1}^{g}\right)-U^{g}\left(j, j+1, y_{j}, y_{j+1}\right)\right] \leq 4+4 Q_{2}, \\
\sum_{j=i_{1}}^{i_{q}-1}[ & \left.U^{g}\left(j, j+1, z_{j}^{g}, z_{j+1}^{g}\right)-U^{g}\left(j, j+1, h_{j}, h_{j+1}\right)\right] \leq 4+4 Q_{2}, \\
& \sum_{j=0}^{N-1}\left[U^{g}\left(j, j+1, z_{j}^{g}, z_{j+1}^{g}\right)-U^{g}\left(j, j+1, y_{j}, y_{j+1}\right)\right] \\
= & \sum_{j=0}^{i_{1}-1}\left[U^{g}\left(j, j+1, z_{j}^{g}, z_{j+1}^{g}\right)-U^{g}\left(j, j+1, y_{j}, y_{j+1}\right)\right] \\
+ & \sum_{j=i_{1}}^{i_{q}-1}\left[U^{g}\left(j, j+1, z_{j}^{g}, z_{j+1}^{g}\right)-U^{g}\left(j, j+1, h_{j}, h_{j+1}\right)\right] \\
+ & \sum_{j=i_{1}}^{i_{q}-1}\left[U^{g}\left(j, j+1, h_{j}, h_{j+1}\right)-U^{g}\left(j, j+1, y_{j}, y_{j+1}\right)\right] \\
+ & \sum_{j=i_{q}}^{N-1}\left[U^{g}\left(j, j+1, z_{j}^{g}, z_{j+1}^{g}\right)-U^{g}\left(j, j+1, y_{j}, y_{j+1}\right)\right]
\end{aligned}
$$

$\leq \sum_{j=0}^{i_{1}-1}\left[U^{g}\left(j, j+1, z_{j}^{g}, z_{j+1}^{g}\right)-U^{g}\left(j, j+1, y_{j}, y_{j+1}\right)\right]+2\left(4+4 Q_{2}\right)-(q-1)$.

This completes the proof of the lemma.

Lemma 4.5. Assume that $g \in U, z \in K|z| \leq M$ and $y:[0, \infty) \rightarrow K$ is an a.c. function which satisfies

$$
\limsup _{t \rightarrow \infty}|y(t)|>Q_{1}
$$

Then

$$
I^{g}(0, T, y)-I^{g}\left(0, T, Z^{g}\right) \rightarrow \infty \text { as } T \rightarrow \infty
$$


Proof. There are two cases: a) $\limsup _{i \rightarrow \infty}|y(i)|>M_{2}$; b) $\lim \sup _{i \rightarrow \infty}|y(i)|$ $\leq M_{2}$ where $i$ is an integer. Consider the case a). It follows from Lemma $4.4,(4.12)$ that

$$
I^{g}(0, q, y)-I^{g}\left(0, q, Z^{g}\right) \rightarrow \infty \text { as an integer } q \rightarrow \infty .
$$

Let $T>0$. There exists an integer $q(T) \geq 0$ such that

$$
q(T)<T \leq q(T)+1 .
$$

By Assumption (Aiii) and (4.14)

$$
\begin{gathered}
I^{g}(q(T), T, y) \geq-a, I^{g}\left(q(T), T, Z^{g}\right)=I^{g}\left(q(T), q(T)+1, Z^{g}\right) \\
-I^{g}\left(T, q(T)+1, Z^{g}\right) \leq Q_{0}+a .
\end{gathered}
$$

Together with (4.34) these relations imply that

$$
\begin{gathered}
I^{g}(0, T, y)-I^{g}\left(0, T, Z^{g}\right) \geq I^{g}(0, q(T), y) \\
-I^{g}\left(0, q(T), Z^{g}\right)-Q_{0}-2 a \rightarrow \infty \text { as } T \rightarrow \infty .
\end{gathered}
$$

Consider the case $\mathrm{b}$ ). There exists an integer $i_{0} \geq 2$ such that

$$
|y(i)| \leq M_{2}+2^{-1} \text { for all integers } i \geq i_{0} .
$$

By (4.37), (4.32), (4.4) and the definition of $Q_{1}$ (see (4.5))

$$
\sum_{i=0}^{N}\left[I^{g}(i, i+1, y)-U^{g}(i, i+1, y(i), y(i+1))\right] \rightarrow \infty \text { as } N \rightarrow \infty .
$$

Define a sequence $\left\{d_{i}\right\}_{i=i_{0}}^{\infty} \subset K$ as follows

$$
d_{i_{0}}=z, d_{i}=y(i) \text { for all integers } i>i_{0} .
$$

By Lemma 4.1 and the definition of $\left\{d_{i}\right\}_{i=i_{0}}^{\infty}$ for any integer $N \geq i_{0}+1$

$$
\begin{gathered}
\sum_{i=i_{0}+1}^{N}\left[U^{g}(i, i+1, y(i), y(i+1))-U^{g}\left(i, i+1, z_{i}^{g}, z_{i+1}^{g}\right)\right]= \\
\sum_{i=i_{0}}^{N}\left[U^{g}\left(i, i+1, d_{i}, d_{i+1}\right)-U^{g}\left(i, i+1, z_{i}^{g}, z_{i+1}^{g}\right)\right]+U^{g}\left(i_{0}, i_{0}+1, z_{i_{0}}^{g}, z_{i_{0}+1}^{g}\right) \\
-U^{g}\left(i_{0}, i_{0}+1, z, y\left(i_{0}+1\right)\right) \geq-4-4 Q_{2}+ \\
U^{g}\left(i_{0}, i_{0}+1, z_{i_{0}}^{g}, z_{i_{0}+1}^{g}\right)-U^{g}\left(i_{0}, i_{0}+1, z, y\left(i_{0}+1\right)\right) .
\end{gathered}
$$

Together with (4.28), (4.12) this implies that

$$
\sum_{i=0}^{N}\left[I^{g}(i, i+1, y)-I^{g}\left(i, i+1, Z^{g}\right)\right] \rightarrow+\infty \text { as } N \rightarrow \infty .
$$

Let $T>0$. There exists an integer $q(T) \geq 0$ satisfying (4.35). Clearly (4.36) holds. (4.33) now follows from (4.36) and (4.39). The lemma is proved. 
Lemma 4.6. Let $g \in U, z \in K,|z| \leq M$ and let $y:[0, \infty) \rightarrow K$ be an a.c. function. Then one of the relations below holds:

(i) $I^{g}(0, T, y)-I^{g}\left(0, T, Z^{g}\right) \rightarrow \infty$ as $T \rightarrow \infty$;

(ii) $\sup \left\{\left|I^{g}(0, T, y)-I^{g}\left(0, T, Z^{g}\right)\right|: T \in(0, \infty)\right\}<\infty$.

Proof. By Lemma 4.5 we may assume that $\limsup _{t \rightarrow \infty}|y(t)| \leq Q_{1}$. There exists an integer $i_{0}>0$ such that

$$
|y(t)| \leq Q_{1}+2^{-1}, t \in\left[i_{0}, \infty\right)
$$

Fix an integer $i>i_{0}$. By Corollary 2.1 there exists an a.c. function $\bar{y}$ : $[i-1, \infty) \rightarrow K$ such that

$$
\bar{y}(i-1)=z, \bar{y}(t)=y(t), t \in[i, \infty), I^{g}(i-1, i, \bar{y})=U^{g}(i-1, i, z, y(i)) .
$$

(4.7), (4.41), (4.40), (4.5) imply that $\left|U^{g}(i-1, i, z, y(i))\right| \leq Q_{2}$. It follows from this relation, (4.41), Lemma 4.2 and Assumption (Aiii) that for each $T>i$

$$
\begin{aligned}
& I^{g}(i, T, y)-I^{g}\left(i, T, Z^{g}\right)=I^{g}(i-1, T, \bar{y})-I^{g}\left(i-1, T, Z^{g}\right) \\
- & I^{g}(i-1, i, \bar{y})+I^{g}\left(i-1, i, Z^{g}\right) \geq-4-4 Q_{2}-Q_{0}-2 a \\
- & I^{g}(i-1, i, \bar{y})+I^{g}\left(i-1, i, Z^{g}\right) \geq-4-5 Q_{2}-Q_{0}-3 a .
\end{aligned}
$$

(4.42) holds for each integer $i>i_{0}$ and each $T>i$.

Let $S>i_{0}+1, T>S+1$. There exists an integer $i>i_{0}+1$ such that $i-1 \leq S<i$. Clearly (4.42) holds. By Assumption (Aiii) and (4.14)

$$
I^{g}(S, i, y) \geq-a, I^{g}\left(S, i, Z^{g}\right)=I^{g}\left(i-1, i, Z^{g}\right)-I^{g}\left(i-1, S, Z^{g}\right) \leq Q_{0}+a .
$$

Together with (4.42) this implies that

$$
\begin{aligned}
I^{g}(S, T, y)-I^{g}\left(S, T, Z^{g}\right) & =I^{g}(i, T, y)-I^{g}\left(i, T, Z^{g}\right)+I^{g}(S, i, y)-I^{g}\left(S, i, Z^{g}\right) \\
& \geq-4-5 Q_{2}-2 Q_{0}-5 a .
\end{aligned}
$$

We established (4.43) for each $S>i_{0}+1$ and each $T>S+1$.

Assume that (ii) does not hold. It follows from (4.14), Assumption (Aiii) and (4.43) which holds for each $S>i_{0}+1, T>S+1$ that

$$
\inf \left\{I^{g}(0, T, y)-I^{g}\left(0, T, Z^{g}\right): T \in(0, \infty)\right\}>-\infty .
$$

Therefore $\sup \left\{I^{g}(0, T, y)-I^{g}\left(0, T, Z^{g}\right): T \in(0, \infty)\right\}=\infty$. By Assumption (Aiii) and (4.14) $\sup \left\{I^{g}(0, i, y)-I^{g}\left(0, i, Z^{g}\right): i=1,2, \ldots\right\}=\infty$. Together with (4.43) which holds for each $S>i_{0}+1, T>S+1$ this implies (i). The lemma is proved. 
Lemma 4.7. Assume that $K=R^{n}, g \in U, z \in K,|z| \leq M, 0 \leq T_{1}<T_{2}$. Then

$$
I^{g}\left(T_{1}, T_{2}, Z^{g}\right)=U^{g}\left(T_{1}, T_{2}, Z^{g}\left(T_{1}\right), Z^{g}\left(T_{2}\right)\right) .
$$

Proof. Let us assume the converse. Fix a number

$$
\epsilon \in\left(0,8^{-1}\left[I^{g}\left(T_{1}, T_{2}, Z^{g}\right)-U^{g}\left(T_{1}, T_{2}, Z^{g}\left(T_{1}\right), Z^{g}\left(T_{2}\right)\right)\right]\right.
$$

and an integer $q_{0}>T_{2}+5$. By Corollary 2.1 there exists an a.c. function $y:\left[T_{1}, T_{2}\right] \rightarrow K$ such that

$$
y\left(T_{i}\right)=Z^{g}\left(T_{i}\right), i=1,2, \quad I^{g}\left(T_{1}, T_{2}, y\right)=U^{g}\left(T_{1}, T_{2}, Z^{g}\left(T_{1}\right), Z^{g}\left(T_{2}\right)\right) .
$$

It follows from $(4.10),(4.11),(4.12)$ and Proposition 2.7 that there exists an integer $k>2 q_{0}+4$ for which

$$
\begin{gathered}
\left|U^{g}\left(i, i+1, Z^{g}(i), Z^{g}(i+1)\right)-U^{g}\left(i, i+1, Z_{k}^{g}(i), Z_{k}^{g}(i+1)\right)\right| \leq \\
\left(2 q_{0}+1\right)^{-1} \epsilon, i=0, \ldots 2 q_{0}+1, \\
\mid U^{g}\left(q_{0}, q_{0}+1, Z^{g}\left(q_{0}\right), Z_{k}^{g}\left(q_{0}+1\right)\right)- \\
U^{g}\left(q_{0}, q_{0}+1, Z_{k}^{g}\left(q_{0}\right), Z_{k}^{g}\left(q_{0}+1\right)\right) \mid \leq\left(2 q_{0}+1\right)^{-1} \epsilon .
\end{gathered}
$$

By Corollary 2.1 and (4.45) there exists an a.c. function $x:[0, k] \rightarrow K$ such that

$$
x(t)=Z^{g}(t), t \in\left[0, T_{1}\right] \cup\left[T_{2}, q_{0}\right], x(t)=y(t), t \in\left[T_{1}, T_{2}\right],
$$

$x(t)=Z_{k}^{g}(t), t \in\left[q_{0}+1, k\right], I^{g}\left(q_{0}, q_{0}+1, x\right)=U^{g}\left(q_{0}, q_{0}+1, x\left(q_{0}\right), x\left(q_{0}+1\right)\right)$.

It follows from (4.48), (4.9) that

$$
I^{g}(0, k, x) \geq I^{g}\left(0, k, Z_{k}^{g}\right) .
$$

By (4.48), (4.9), (4.12), (4.46), (4.47) and (4.44)

$$
\begin{gathered}
I^{g}(0, k, x)-I^{g}\left(0, k, Z_{k}^{g}\right)=I^{g}\left(0, q_{0}+1, x\right)-I^{g}\left(0, q_{0}+1, Z_{k}^{g}\right)= \\
\left(I^{g}\left(0, q_{0}, x\right)-I^{g}\left(0, q_{0}, Z^{g}\right)\right)+\left(I^{g}\left(0, q_{0}, Z^{g}\right)-I^{g}\left(0, q_{0}, Z_{k}^{g}\right)\right)+I^{g}\left(q_{0}, q_{0}+1, x\right) \\
-I^{g}\left(q_{0}, q_{0}+1, Z_{k}^{g}\right) \leq I^{g}\left(T_{1}, T_{2}, y\right)-I^{g}\left(T_{1}, T_{2}, Z^{g}\right) \\
+\sum_{i=0}^{q_{0}-1}\left[U^{g}\left(i, i+1, Z^{g}(i), Z^{g}(i+1)\right)-U^{g}\left(i, i+1, Z_{k}^{g}(i), Z_{k}^{g}(i+1)\right)\right] \\
+U^{g}\left(q_{0}, q_{0}+1, Z^{g}\left(q_{0}\right), Z_{k}^{g}\left(q_{0}+1\right)\right)-U^{g}\left(q_{0}, q_{0}+1, Z_{k}^{g}\left(q_{0}\right), Z_{k}^{g}\left(q_{0}+1\right)\right) \leq
\end{gathered}
$$




$$
I^{g}\left(T_{1}, T_{2}, y\right)-I^{g}\left(T_{1}, T_{2}, Z^{g}\right)+\epsilon .
$$

It follows from this relation, (4.44), (4.45) that

$$
I^{g}(0, k, x)-I^{g}\left(0, k, Z_{k}^{g}\right) \leq I^{g}\left(T_{1}, T_{2}, y\right)-I^{g}\left(T_{1}, T_{2}, Z^{g}\right)+\epsilon<-\epsilon .
$$

This is contradictory to (4.49). The obtained contradiction proves the lemma.

Proof of Theorem 1.1. At the begining of Section 4 for each $f \in \mathfrak{M}$ and each $M>2|\bar{z}|$ we constructed a neighborhood $U$ of $f$ in $\mathfrak{M}$ and for each $g \in U$ and each $z \in K$ satisfying $|z| \leq M$ we defined a.c. functions $Z^{g}$ : $[0, \infty) \rightarrow K, Z_{q}^{g}:[0, q] \rightarrow K, q=1,2, \ldots$ satisfying (4.9)-(4.15). Clearly an a.c. function $Z^{f}:[0, \infty) \rightarrow K$ was defined for every $f \in \mathfrak{M}$ and every $z \in K$. By Lemmas 4.5,4.6 for each $f \in \mathfrak{M}$ and each $z \in K$ the function $Z^{f}$ is $(f)$-good and Assertion 1 of Theorem 1.1 holds.

Assertion 2 of Theorem 1.1 follows from (4.15) which holds for every $g \in U$ ( $U$ is a neighborhood of $f$ in $\mathfrak{M}$ ) and each $z \in K$ satisfying $|z| \leq M$.

Assertion 3 of Theorem 1.1 follows from Lemma 4.3. Lemma 4.7 implies Assertion 4 of Theorem 1.1. Theorem 1.1 is proved.

Theorem 1.2 follows from Lemma 4.5.

Proof of Theorem 1.3. Fix $\bar{z} \in K$. By Proposition 2.6 there exists a neighborhood $U_{0}$ of $f$ in $\mathfrak{M}$ and a number

$M_{0} \geq \sup \left\{\left|U^{g}\left(T_{1}, T_{2}, y, z\right)\right|: g \in U_{0}, T_{1} \in[0, \infty), T_{2} \in\left[T_{1}+c, T_{1}+2 c+2\right]\right.$,

$$
y, z \in K,|y|,|z| \leq 2|\bar{z}|+1\} .
$$

By Proposition 2.3 we may assume without loss of generality that

(4.51) $\inf \left\{U^{g}\left(T_{1}, T_{2}, y, z\right): g \in \mathfrak{M}, T_{1} \in[0, \infty), T_{2} \in\left[T_{1}+c, T_{1}+2 c+2\right]\right.$,

$$
\left.y, z \in K,|y|+|z| \geq M_{1}\right\}>2 M_{0}+1 .
$$

There exists a neighborhood $U_{1}$ of $f$ in $\mathfrak{M}$ and a number $S_{1}$ such that

$$
\begin{gathered}
U_{1} \subset U_{0}, S_{1}>M_{1} \text { and Proposition } 3.2 \text { holds with } \\
M_{3}=M_{2}+2, M_{4}=S_{1}, V=U_{1}, c_{1}=c, c_{2}=2 c+2 .
\end{gathered}
$$

By Proposition 2.6 there exist a neighborhood $U$ of $f$ in $\mathfrak{M}$ and a number $M_{3}>0$ such that

$$
\begin{aligned}
& U \subset U_{1}, M_{3}>\sup \left\{\left|U^{g}\left(T_{1}, T_{2}, y, z\right)\right|: g \in U, T_{1} \in[0, \infty),\right. \\
& \left.T_{2} \in\left[T_{1}+c, T_{1}+2 c+2\right], y, z \in K,|y|,|z| \leq S_{1}\right\} .
\end{aligned}
$$


By Proposition 2.3 there exist $S>S_{1}+1$ such that $|v(t)| \leq S, t \in\left[T_{1}, T_{2}\right]$ for each $g \in \mathfrak{M}$, each $T_{1} \in[0, \infty), T_{2} \in\left[T_{1}+c, T_{1}+2 c+2\right]$ and each a.c. function $v:\left[T_{1}, T_{2}\right] \rightarrow K$ satisfying $I^{g}\left(T_{1}, T_{2}, v\right) \leq 2 M_{3}+2 M_{2}+2$.

Assume that $g \in U, T_{1} \in[0, \infty), T_{2} \geq c+T_{1}$. We will show that property (i) holds.

Let $x, y \in K,|x|,|y| \leq M_{1}$ and let $v:\left[T_{1}, T_{2}\right] \rightarrow K$ be an a.c. function which satisfies

$$
v\left(T_{1}\right)=x, v\left(T_{2}\right)=y, I^{g}\left(T_{1}, T_{2}, v\right) \leq U^{g}\left(T_{1}, T_{2}, x, y\right)+M_{2} .
$$

There is a natural number $p$ such that $p c \leq T_{2}-T_{1}<(p+1) c$. Set $T=p^{-1}\left(T_{2}-T_{1}\right)$. Clearly $T \in[c, 2 c]$. By (4.54) and Corollary 2.1

$$
\begin{gathered}
\sum_{i=0}^{p-1}\left[U^{g}\left(T_{1}+i T, T_{1}+(i+1) T, v\left(T_{1}+i T\right), v\left(T_{1}+(i+1) T\right)\right)\right. \\
\left.-U^{g}\left(T_{1}+i T, T_{1}+(i+1) T, y_{i}, y_{i+1}\right)\right] \leq M_{2}
\end{gathered}
$$

for each sequence $\left\{y_{i}\right\}_{i=0}^{p} \subset K$ satisfying $y_{0}=v\left(T_{1}\right), y_{p}=v\left(T_{2}\right)$. It follows from this, (4.52), (4.54) and Proposition 3.2 that

$$
\left|v\left(T_{1}+i T\right)\right| \leq S_{1}, i=0, \ldots p
$$

By this relation and (4.54), (4.53) for $i=0, \ldots p-1$

$$
\begin{gathered}
I^{g}\left(T_{1}+i T, T_{1}+(i+1) T, v\right) \leq \\
U^{g}\left(T_{1}+i T, T_{1}+(i+1) T, v\left(T_{1}+i T\right), v\left(T_{1}+(i+1) T\right)\right)+M_{2}<M_{3}+M_{2} .
\end{gathered}
$$

It follows from this relation and the definition of $S$ that

$$
|v(t)| \leq S, t \in\left[T_{1}, T_{2}\right]
$$

Therefore property (i) holds. Analogously to this we can show that property (ii) holds. The theorem is proved.

\section{REFERENCES}

1. B. D. O. Anderson and J. B. Moore, Linear optimal control, Prentice-Hall, Englewood Cliffs, New Jersey, 1971.

2. Z. Artstein and A. Leizarowitz, Tracking periodic signals with overtaking criterion, IEEE Trans. Automat. Control AC 30 (1985), 1122-1126.

3. S. Aubry and P. Y. Le Daeron, The discrete Frenkel-Kontorova model and its extensions. I. Exact Results for the ground-states, Phys. D 8 (1983), 381-422.

4. L. D. Berkovitz, Lower semicontinuity of integral functionals, Trans. Amer. Math. Soc. 192 (1974), 51-57.

5. W. A. Brock and A. Haurie, On existence of overtaking optimal trajectories over an infinite horizon, Math. Oper Res. 1 (1976), 337-346. 
6. D. A. Carlson, The existence of catching-up optimal solutions for a class of infinite horizon optimal control problems with time delay, SIAM J. Control Optim. 28 (1990), 402-422.

7. B. D. Coleman, M. Marcus and V. J. Mizel, On the thermodynamics of periodic phases, Arch. Rational Mech. Anal. 117 (1992), 321-347.

8. D. Gale, On optimal development in a multisector economy, Rev. Econom. Stud. 34 (1967), 1-19.

9. H. Halkin, Necessary conditions for optimal control problems with infinite horizon, Econometrica 42 (1974), 267-273.

10. A. Leizarowitz, Infinite horizon autonomous systems with unbounded cost, Appl. Math. Optim. 13 (1985), 19-43.

11. A. Leizarowitz, Existence of overtaking optimal trajectories for problems with convex integrands, Math. Oper. Res. 10 (1985), 450-461.

12. A. Leizarowitz and V. J. Mizel, One-dimensional infinite horizon variational problems arising in continuum mechanics, Arch. Rational Mech. Anal. 106 (1989), 161-194.

13. J. Moser, Minimal solutions of variational problems on a torus, Ann. Inst. H. Poincaré Anal. Non Lináire 3 (1986), 229-272.

14. R. T. Rockafellar, Saddle points of Hamiltonian systems in convex problem of Lagrange, Journal Optim. Theory Appl. 12 (1973), 367-389.

15. C. C. von Weizsacker, Existence of optimal programs of accumulation for an infinite horizon, Rev. Econom. Stud. 32 (1965), 85-104.

16. A. J. Zaslavski, Ground states in Frenkel-Kontorova model, Math. USSR Izvestiya 29 (1987), 323-354.

17. A. J. Zaslavski, The existence and structure of extremals for a class of second order infinite horizon variational problems, J. Math. Anal. Appl. 194 (1995), 660-696.

18. A. J. Zaslavski, Structure of extremals for one-dimensional variational problems arising in continuum mechanics, J. Math. Anal. Appl. 198 (1996), 893-921.

19. A. J. Zaslavski, Dynamic properties of optimal solutions of variational problems, Nonlinear Anal. 27 (1996), 895-931.

20. A. J. Zaslavski, Turnpike theorem for a class of differential inclusions arising in economic dynamics, Optimization 42 (1997), 139-168.

\section{Department of Mathematics}

TECHnion-IsRael Institute of TeChnology

32000 HAIFA, ISRAEL

E-mail address: ajzasl@techunix.technion.ac.il 


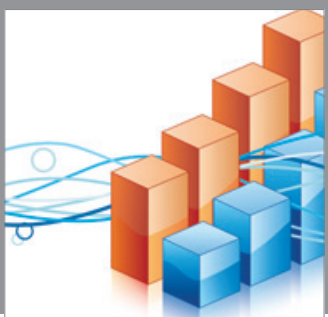

Advances in

Operations Research

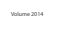

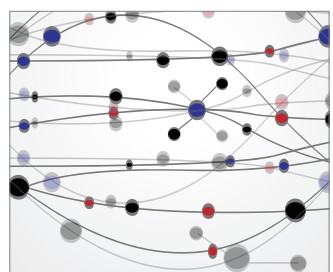

\section{The Scientific} World Journal
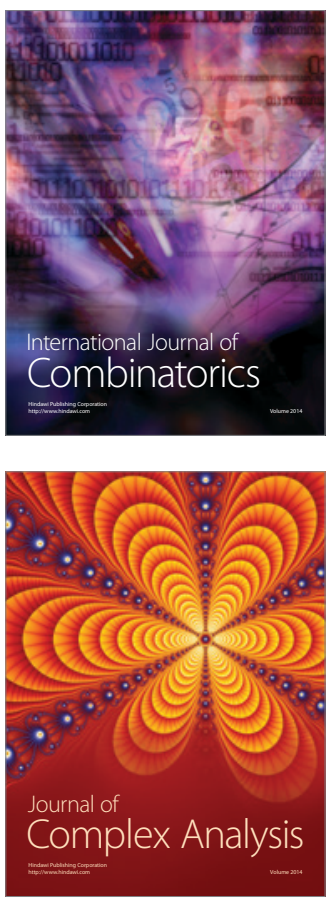

International Journal of

Mathematics and

Mathematical

Sciences
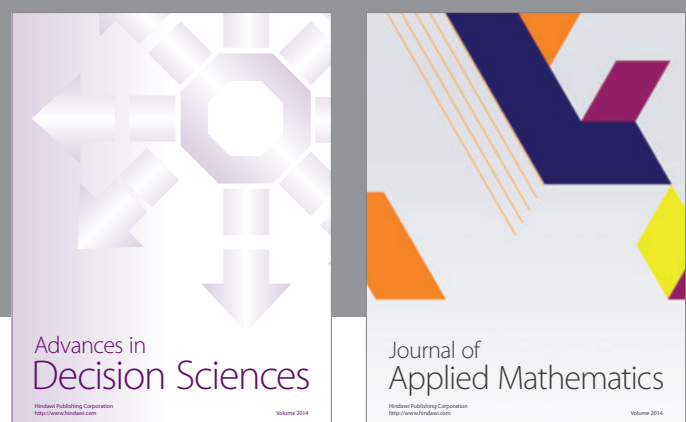

Journal of

Applied Mathematics
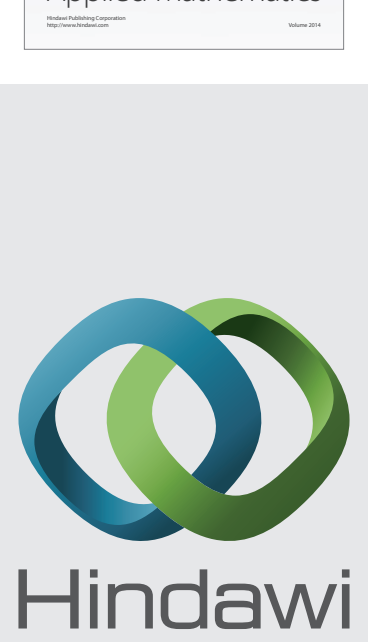

Submit your manuscripts at http://www.hindawi.com
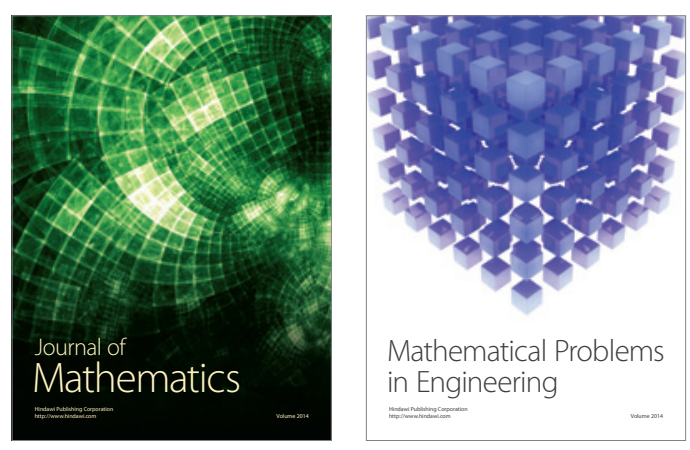

Mathematical Problems in Engineering
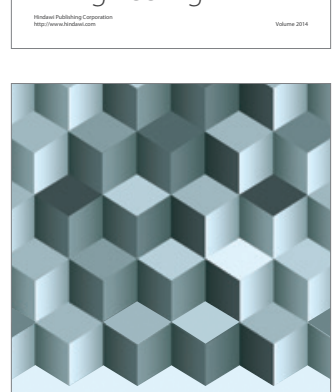

Journal of

Function Spaces
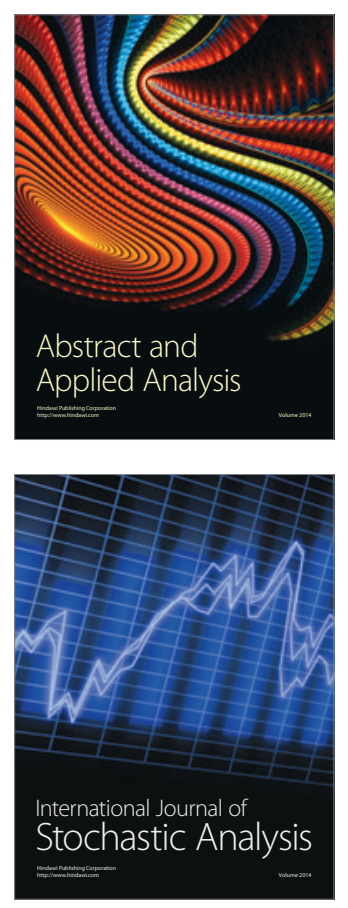

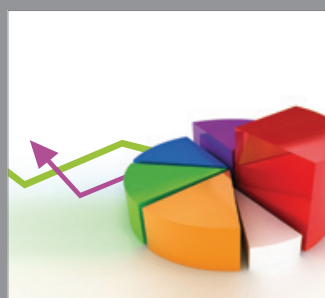

ournal of

Probability and Statistics

Promensencen
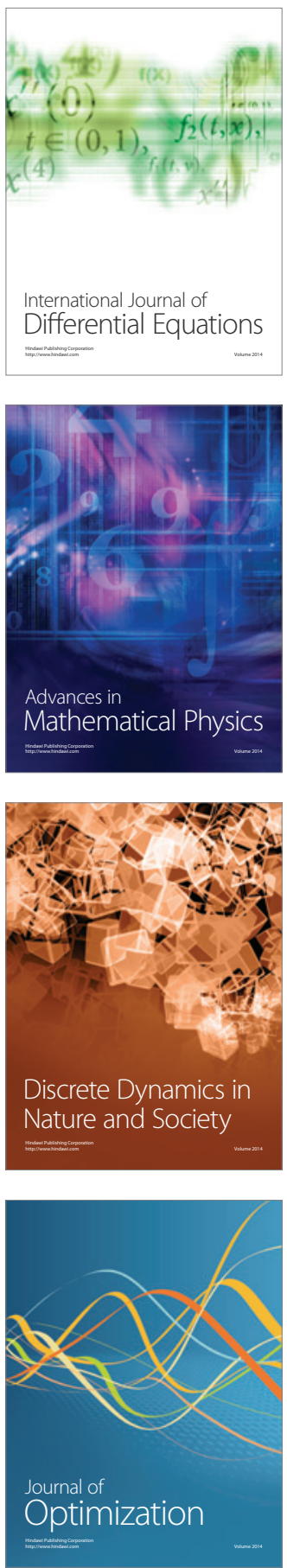